The Astronomical Journal, 122:1213-1237, 2001 September

\title{
HIGH-RESOLUTION MID-INFRARED IMAGING OF INFRARED-LUMINOUS STARBURST GALAXIES ${ }^{1}$
}

\author{
B. T. Soifer, ${ }^{2}$ G. Neugebauer, K. Matthews, E. Egami, and A. J. Weinberger ${ }^{3}$ \\ Palomar Observatory, California Institute of Technology, Mail Stop 320-47, Pasadena, CA 91125; bts@mop.caltech.edu, gxn@mop.caltech.edu, \\ kym@caltech.edu, egami@mop.caltech.edu, alycia@astro.ucla.edu \\ M. RESSLER \\ Jet Propulsion Laboratory, California Institute of Technology, Mail Stop 169-506, 4800 Oak Grove Drive, Pasadena, CA 91109; \\ ressler@cougar.jpl.nasa.gov \\ N. Z. SCOVILLE \\ Division of Physics, Mathematics, and Astronomy, California Institute of Technology, Mail Stop 105-24, Pasadena, CA 91125; nzs@astro.caltech.edu \\ S. R. Stolovy \\ SIRTF Science Center, California Institute of Technology, Mail Stop 314-6, Pasadena, CA 91125; stolovy@ipac.caltech.edu \\ J. J. CONDON \\ National Radio Astronomy Observatory, 520 Edgemont Road, Charlottesville, VA 22903; jcondon@nrao.edu \\ AND \\ E. E. BECKLIN \\ Department of Physics and Astronomy, UCLA, Los Angeles, CA 90095; becklin@astro.ucla.edu \\ Received 2001 March 25; accepted 2001 June 6
}

\begin{abstract}
Observations for seven infrared-luminous starburst galaxies are reported in the mid-infrared from 8 to $18 \mu \mathrm{m}$ using the Keck telescopes with spatial resolution approaching the diffraction limit. All of the galaxies observed show evidence of strong interactions based on optical morphologies. For these galaxies, a substantial fraction, usually more than $50 \%$, of the infrared luminosity is generated in regions ranging in sizes from $100 \mathrm{pc}$ to $1 \mathrm{kpc}$. Nuclear starbursts often dominate the infrared luminosity, but this is not always true. In some galaxies, most notably NGC 6090, substantial infrared luminosity greatly in excess of the nuclear luminosity is generated in regions associated with the physical interaction between two galaxies. The radio emission is a good tracer of the location of high-luminosity young stars. The visual/ultraviolet radiation output of the nearby star-forming galaxies is dominated by emission from regions that are generally not producing the copious infrared luminosity of the systems. As seen in comparing the mid-infrared and near-infrared images of the galaxies observed here, the regions of highinfrared luminosity in local galaxies are significantly smaller than the galaxies as a whole. The integrated spectral energy distributions (SEDs) of these galaxies are very different from the SEDs of the regions of star formation. If the SEDs of star-forming regions in these galaxies reflect the SEDs that would be found in forming galaxies at high redshift, we would expect the distant galaxies to be dominated by the mid- and far-infrared luminosity output far more than the integrated luminous output of nearby starburst galaxies would suggest.
\end{abstract}

Key words: galaxies: general — galaxies: starburst — infrared radiation

\section{INTRODUCTION}

Starburst galaxies have long been known to be copious emitters of infrared luminosity. From early observations of nearby starburst systems such as M82 and NGC 253 (see e.g., Kleinmann \& Low 1970; Rieke \& Low 1972; Harper \& Low 1973), it has been clear that the bulk of the energy of these systems emerges in the thermal infrared. Analysis of the IRAS Bright Galaxy Sample showed that $30 \%$ of the energy output in the local universe emerges in the mid- and far-infrared (5-200 $\mu \mathrm{m}$; Soifer \& Neugebauer 1991), and the

\footnotetext{
${ }^{1}$ Some of the data presented herein were obtained at the W. M. Keck Observatory, which is operated as a scientific partnership among the California Institute of Technology, the University of California, and the National Aeronautics and Space Administration. The Observatory was made possible by the generous financial support of the W. M. Keck Foundation.

${ }^{2}$ Also at SIRTF Science Center, California Institute of Technology, Mail Code 314-6, Pasadena, CA 91125.

${ }^{3}$ Current address: Department of Physics and Astronomy, 156-205, UCLA, Los Angeles, CA 90095.
}

bulk of this luminosity is due to star formation in these galaxies. Recently, the idea that the bulk of the radiant energy emitted in the universe has been emitted at infrared wavelengths has been raised through analysis of the farinfrared background as measured by the COBE satellite (Hauser et al. 1998; Puget et al. 1996). Further, deep submillimeter surveys have suggested that much of this luminosity is being generated in forming galaxies at redshifts of $z \geq 2$ (e.g., Eales et al. 2000).

Infrared-luminous starbursts in the local universe afford us the best opportunity to study the processes by which such systems form and evolve and provide detailed examples where we can test physical models of such systems. The IR $A S$ all-sky survey (IRAS Point Source Catalog, Version 2 1989) has provided a unique database from which many such systems have been chosen for detailed examination.

A wide variety of observational techniques have been employed to probe infrared-luminous starburst galaxies, ranging from optical spectroscopy (e.g., Kim et al. 1995; Veilleux et al. 1995) and mid-infrared imaging and spectroscopy (Keto et al. 1997; Miles et al. 1996; Roche et al. 1991; 
Dudley 1999) to centimeter radio continuum imaging (Condon et al. 1990, 1991, hereafter C91). The nuclear environments of starburst galaxies have been effectively imaged with high spatial resolution in the near-infrared by, e.g., Scoville et al. (2000, hereafter S00), Dinshaw et al. (1999), and Alfonso-Herrero et al. (2000, 2001).

Because dust responds through its thermal emission instantaneously to the radiation field in which it is embedded, thermal radiation by dust is the ideal way to trace the current location of the dominant luminosity sources, i.e., the most luminous stars, in starbursts. In very dusty systems, such as those known from IRAS observations to be luminous starbursts, high spatial resolution imaging in the thermal infrared can address the location and extent of the current star formation in these galaxies unimpeded by foreground extinction. How the thermal dust emission is related to the other tracers of star formation is important for utilizing these tracers in more distant galaxies.

IRAS measured the total bolometric luminosity from these galaxies (e.g., Soifer et al. 1987). The spatial resolution of those observations, $\sim 1^{\prime}-2^{\prime}$, was, however, inadequate to locate the emission within the galaxies. Because the midinfrared wavelengths $(8-25 \mu \mathrm{m})$ carry a significant fraction of the total bolometric luminosity in infrared bright galaxies, ranging from $\sim 10 \%$ to greater than $30 \%$ of the 8-1000 $\mu \mathrm{m}$ luminosity, observations at these wavelengths with a resolution $\leq 1^{\prime \prime}$ have the potential to directly trace the locations where the most luminous stars are forming in these systems. With a diffraction limit of 0".24 FWHM at 10 $\mu \mathrm{m}$, the Keck telescope provides a substantial improvement in spatial resolution over previous mid-infrared observations, probing the distribution of the thermal emission at the $100-300$ pc scale in luminous starbursts at distances of 40-120 Mpc.

In this paper, we report imaging observations from 8-18 $\mu \mathrm{m}$ of a sample of highly luminous starburst galaxies at spatial resolutions of $0^{\prime \prime} 3-0$ ". 6 . These data provide the highest spatial resolution yet achieved of the thermal emission from these systems and trace the spatial distribution of the emergent luminosity in these systems. For purposes of establishing luminosities and physical distances in these galaxies, we adopt $H_{0}=75 \mathrm{~km} \mathrm{~s}^{-1} \mathrm{Mpc}^{-1}$.

\section{THE SAMPLE}

The objects observed were taken from the IRAS Bright Galaxy Sample (BGS, Soifer et al. 1987, 1989). The basic information for the seven objects in the sample is given in Table 1. Since the goal of this study is to understand luminous starburst galaxies, systems having Seyfert-like spectra were excluded. With this selection criterion, there are
25 objects in the BGS in the luminosity range $11.4 \leq$ $\log \left(L_{\mathrm{bol}}\left[L_{\odot}\right]\right) \leq 11.9$; seven were observed. These seven objects are among the closest highly infrared-luminous starburst galaxies. Six of the seven objects observed have clear evidence of strong interactions/mergers from optical images. The only object without such evidence, Mrk 331 (= UGC 12812), has several close companions. In this luminosity range, $60 \%-70 \%$ of objects are found in strongly interacting or merging systems (Sanders et al. 1988; Sanders $\&$ Mirabel 1996). The objects were selected to be detectable from the ground at $12 \mu \mathrm{m}$, so that there is a bias toward brighter $12 \mu \mathrm{m}$ flux densities in these galaxies, as measured by $I R A S$, compared with the galaxies in the same luminosity range in the BGS. Three of the galaxies in the sample, IC 1623 (=VV 114), NGC 3690/IC 694 (=Arp 299), and NGC 1614 (=Arp 186) are the three brightest objects at 12 $\mu \mathrm{m}$ in the above sample. This selection of brighter $12 \mu \mathrm{m}$ flux density appears to be the major bias in our sample, as compared with the total BGS sample for this luminosity range.

\section{OBSERVATIONS AND DATA REDUCTION}

The observations were made using the MIRLIN midinfrared camera (Ressler et al. 1994) at the $\mathrm{f} / 40$ bent Cassegrain visitor port of the Keck II telescope and the imaging mode of the long wavelength spectrograph (LWS; Jones \& Puetter 1993) at the $f / 25$ forward Cassegrain focus of the Keck I telescope. In general, only observations where the FWHM of the PSF was less than 1".0 were used. Because of the variability, the seeing conditions are described throughout the text when specific objects are discussed.

The MIRLIN camera uses a $128 \times 128 \mathrm{Si}$ :As array with a plate scale of 0.138 pixel $^{-1}$ for a total field of view of $17^{\prime \prime} \times 17^{\prime \prime}$. At each wavelength, the observing procedure was the same. A secondary with a square wave chop of amplitude $6^{\prime \prime}$ in the north-south or east-west direction at $4 \mathrm{~Hz}$ was employed for fast beam switching. The frames sampling each chop position were co-added separately in hardware, resulting in two images. After an interval of approximately a minute, the telescope was nodded perpendicular to the chop direction (east-west or north-south) by 6", and a second pair of images was obtained in order to cancel residuals in the sky and to subtract telescope emission. This procedure was repeated a number of times at each wavelength. The data were reduced by differencing the two images obtained within the chop pairs at each nod location. Then the resulting positive images were co-added, with the positions appropriately adjusted to a common location, to yield an image centered in a field approximately $6^{\prime \prime} \times 6^{\prime \prime}$. Because of the chopper and telescope nod spacings employed for the

TABLE 1

Basic Properties of Observed Galaxies

\begin{tabular}{|c|c|c|c|c|c|}
\hline Name & $z$ & $\begin{array}{c}\log L \\
\left(L_{\mathrm{bol}}\left[L_{\odot}\right]\right)\end{array}$ & $\begin{array}{c}\log M_{\mathbf{H}_{2}} \\
\left(M_{\odot}\right)\end{array}$ & Spectrum (opt/IR) & $\begin{array}{l}\text { Linear Scale } \\
\left(\mathrm{pc} \operatorname{arcsec}^{-1}\right)\end{array}$ \\
\hline VV $114(=\operatorname{Arp} 236) \ldots \ldots$. & 0.0200 & 11.62 & 10.44 & $\mathrm{H} \mathrm{II/PAH}$ & 400 \\
\hline NGC $1614(=\operatorname{Arp} 186) \ldots \ldots \ldots \ldots . . .$. & 0.0159 & 11.62 & 10.03 & $\mathrm{H} \mathrm{II} / \ldots$ & 320 \\
\hline NGC $2623(=\operatorname{Arp} 243) \ldots \ldots \ldots \ldots \ldots$ & 0.0185 & 11.54 & 9.77 & $\ldots /$ PAH & 370 \\
\hline NGC 3690/IC 694 (=Arp 299)........ & 0.0104 & 11.91 & 10.06 & $\mathrm{H} \mathrm{II} / \mathrm{PAH}+\mathrm{Sil}$ & 210 \\
\hline IC 883 (=Arp 193) ..................... & 0.0233 & 11.51 & 9.87 & LINER/PAH & 470 \\
\hline NGC $6090(=$ Mrk 496) .............. & 0.0293 & 11.51 & 10.15 & $\mathrm{H} \mathrm{II} / \ldots$ & 590 \\
\hline UGC $12812(=$ Mrk 331$) \ldots \ldots \ldots \ldots$ & 0.0185 & 11.41 & 10.11 & $\mathrm{H} \mathrm{II/PAH}+\mathrm{Sil}$ & 370 \\
\hline
\end{tabular}


observations, the data are not capable of measuring low surface brightness emission that is extended beyond a $6^{\prime \prime}$ diameter region. In addition, extended emission within a $6^{\prime \prime}$ region with a surface brightness of $5 \mathrm{mJy} \operatorname{arcsec}^{-2}$ is not detectable within these images.

Observations with MIRLIN of VV 114, NGC 1614, NGC 3690, and Mrk 331 were obtained in 1998 March and October. The MIRLIN observations were made under photometric conditions. The observations of the targets were interleaved with observations of nearby bright stars that served as photometric calibration and to establish the point-spread function (PSF) for the observations.

Observations of all the galaxies listed in Table 1 were made with LWS in 1999 October and 2000 January, May, and September. Generally, these observations were made under photometric conditions. Over the course of the observations, the mid-infrared seeing varied from near diffraction limit ( $\sim 0^{\prime \prime} .3$ at $\left.12.5 \mu \mathrm{m}\right)$ to greater than 1".0.

The secondary chopper was set to an amplitude of $5^{\prime \prime}$ at a frequency $\sim 5 \mathrm{~Hz}$ for all the objects except NGC 1614, NGC 2623, and IC 883, where the chopping amplitude was $4 "$. Observations were made in a fashion similar to the MIRLIN observations, except that the telescope nodding was in the same direction as the chopping, and the nodding amplitude was the same as the chopping amplitude. The data were reduced in a manner similar to the MIRLIN data. The pixel scale is $0.08 \mathrm{pixel}^{-1}$.

The filters defining the photometric bands are quite similar in both MIRLIN and LWS, and all have widths about $10 \%$ of the central wavelengths that are listed in Table 2. Details of the filters are given in Soifer et al. (2000). The photometry was calibrated based on observations of four bright stars, $\alpha$ Tau (=HR 1457) $([12.5 \mu \mathrm{m}]=-3.07$ $\mathrm{mag}), \alpha$ Boo (=HR 5340) $([12.5 \mu \mathrm{m}]=-3.15 \mathrm{mag}), \beta$ Peg $(=\mathrm{HR}$ 8775) $([12.5 \mu \mathrm{m}]=-2.55 \mathrm{mag})$, and $\alpha \operatorname{Cet}(=\mathrm{HR}$ 0911) $([12.5 \mu \mathrm{m}]=-1.92 \mathrm{mag})$, whose magnitudes in turn were based on IRAS and Keck data. The magnitudes adopted at the other mid-infrared wavelengths for these stars were within $0.08 \mathrm{mag}$ of these values. The uncertainties in the photometry, based on the internal consistency of the observations, is estimated to be $5 \%$ at $\lambda \leq 17.9 \mu \mathrm{m}$. The flux density corresponding to $0.0 \mathrm{mag}$ (Vega-based) was taken to follow the prescription given in the Explanatory Supple- ment to the IRAS catalogs and atlases (Beichman et al. 1989) and is given in Soifer et al. (2000).

In addition, high spatial resolution observations of two of these galaxies, VV 114 (=Arp 236) and NGC 3690 (=Arp 299) were obtained at $3.2 \mu \mathrm{m}$ with NIRC (Matthews \& Soifer 1994) on the Keck I telescope in 1996 December. Although the background is higher at this wavelength than at shorter wavelengths, observations were made in the standard "stare-and-dither" mode for near-infrared observations. Observations were obtained for Mrk 331 at 2.15 $\mu \mathrm{m}\left(K_{\mathrm{s}}\right)$ and $3.4 \mu \mathrm{m}\left(L^{\prime}\right)$ using the near-infrared camera on the 200 inch $(5 \mathrm{~m})$ Hale telescope. Observations were made in a similar fashion to those on the Keck telescope.

\section{RESULTS AND DISCUSSION FOR INDIVIDUAL GALAXIES}

The basic observational results are presented in Table 2 and Figures 1-7. The observed flux densities, measured from the Keck images in the largest beams feasible for each of the galaxies, are presented in Table 2. All of the galaxies were observed at 11.7 and $12.5 \mu \mathrm{m}$, while most of them were observed at $17.9 \mu \mathrm{m}$. For comparison, the IRAS flux densities at $12 \mu \mathrm{m}$, representing the integrated flux densities for the galaxies, are also presented in Table 2 .

In each image, the locations of the origin and, in some cases, other positions are marked to facilitate comparisons. Because bright stars are not common in the mid-infrared, the a priori astrometric registration of the mid-infrared images with respect to other images is not better than 1 ". The locations of the images in these figures were determined by identifying morphologically similar features and assuming that they are spatially coincident at wavelengths between the radio and near-infrared. In the mid-infrared, morphological features within $\sim 5^{\prime \prime}$ diameters were imaged on a single array, and thus differences on that scale between images at different wavelengths are robust.

In the plots that present the spectral energy distributions (SEDs) for these galaxies, a variety of data are presented. The integrated flux densities at each wavelength are from 2MASS and IRAS data. The large beam Keck data are presented (from Table 2), as well as data from NICMOS (or ground-based images), with the photometry scaled to the same size beams. In addition, to illustrate how the SEDs vary with beam size, we also present multiaperture pho-

TABLE 2

KeCK Mid-Infrared Flux Densities for Starburst GalaXIES ${ }^{a}$

\begin{tabular}{ccccccccc}
\hline \hline Object & $\begin{array}{c}7.9 \mu \mathrm{m} \\
(\mathrm{mJy})\end{array}$ & $\begin{array}{c}8.8 \mu \mathrm{m} \\
(\mathrm{mJy})\end{array}$ & $\begin{array}{c}9.7 \mu \mathrm{m} \\
(\mathrm{mJy})\end{array}$ & $\begin{array}{c}10.3 \mu \mathrm{m} \\
(\mathrm{mJy})\end{array}$ & $\begin{array}{c}11.7 \mu \mathrm{m} \\
(\mathrm{mJy})\end{array}$ & $\begin{array}{c}12.5 \mu \mathrm{m} \\
(\mathrm{mJy})\end{array}$ & $\begin{array}{c}12 \mu \mathrm{m}^{\mathrm{b}} \\
(\mathrm{mJy})\end{array}$ & $\begin{array}{c}17.9 \mu \mathrm{m} \\
(\mathrm{mJy})\end{array}$ \\
\hline VV $114 \mathrm{E} \ldots \ldots \ldots \ldots$. & 457 & 245 & $52 \pm 21$ & $70 \pm 10$ & 237 & 338 & 1100 & $452 \pm 61$ \\
NGC $1614^{\mathrm{c}} \ldots \ldots \ldots \ldots$ & $\ldots$ & $\ldots$ & $\ldots$ & $\ldots$ & 1030 & 1220 & 1400 & $\ldots$ \\
NGC $2623 \ldots \ldots \ldots \ldots$ & $\ldots$ & $\ldots$ & $\ldots$ & $\ldots$ & 151 & 201 & 240 & $\ldots$ \\
NGC $3690 \mathrm{sum}^{\mathrm{d}} \ldots \ldots$. & $\ldots$ & $\ldots$ & $\ldots$ & $\ldots$ & 2390 & 4030 & 3900 & 12650 \\
IC $883 \ldots \ldots \ldots \ldots \ldots$. & $335 \pm 34$ & $\ldots$ & $\ldots$ & $34 \pm 11$ & 130 & $301 \pm 46$ & 260 & $\ldots$ \\
NGC $6090 \mathrm{sum}^{\mathrm{e}} \ldots \ldots$. & $\ldots$ & $\ldots$ & $\ldots$ & $\ldots$ & 167 & 134 & 290 & $356 \pm 40$ \\
Mrk $331 \ldots \ldots \ldots \ldots \ldots$ & $\ldots$ & $\ldots$ & $\ldots$ & $\ldots$ & 280 & 403 & 510 & 685 \\
\hline
\end{tabular}

a The Keck flux densities are in a 4" diameter circular beam unless otherwise noted. The uncertainties are omitted from the table unless the statistical uncertainty in the photometry, based on the noise in the sky, exceeds $10 \%$. The uncertainties quoted are statistical only; photometric uncertainties are on the order of $\pm 10 \%$.

${ }^{\mathrm{b}}$ The $12 \mu \mathrm{m}$ flux densities are from the IRAS data for these objects. The effective beam size is $2^{\prime}$ for the IRAS measurements. The uncertainties in the IRAS data are $5 \%-10 \%$.

${ }^{c}$ At $24.5 \mu \mathrm{m}$ a flux density of $7.0 \mathrm{Jy}$ was measured.

d The flux densities are the sum of the flux densities measured in four separate 4" diameter beams centered on NGC 3690 A, $3690 \mathrm{~B} 1,3690 \mathrm{C}$, and $3690 \mathrm{C}^{\prime}$, and a 2" diameter circular beam centered at NGC $3690 \mathrm{~B} 2$.

e The flux densities in a 6" diameter beam centered on NGC $6090 \mathrm{E}_{\mathrm{S}}$ and a 4 " beam centered on NGC 6090W. 
tometry at the locations where there is significant $12.5 \mu \mathrm{m}$ emission in the Keck images. The beam diameters range from $1^{\prime \prime}$ to $6^{\prime \prime}$, depending on the size and complexity of the source. Photometry is taken from Keck mid-infrared, NICMOS, and ground-based near-infrared data. The details of the small-beam photometry are explained in the corresponding figure captions.

In the following sections, we discuss the observational results for the galaxies separately.

\section{1. $V V 114(=I C 1623=\operatorname{Arp} 236)$}

At a redshift of $c z=6000 \mathrm{~km} \mathrm{~s}^{-1}(80 \mathrm{Mpc}, 400 \mathrm{pc}$ $\left.\operatorname{arcsec}^{-1}\right)$, VV $114(=$ Arp 236) is a highly disturbed system that shows two major centers in optical images, with low surface brightness tails evident over $55^{\prime \prime}(22 \mathrm{kpc})$ in photographic images (Arp 1966). The two centers are aligned east-west and separated by about $20^{\prime \prime}(8 \mathrm{kpc})$. The bolometric luminosity of the system is $\sim 4 \times 10^{11} L_{\odot}$ (Soifer et al. 1987), more than $90 \%$ of which emerges at far-infrared wavelengths.

Previous detailed near-infrared studies of VV 114 have been reported by Knop et al. (1994) and Doyon et al. (1995). High-resolution near-infrared imaging of VV 114 has been reported by S00. Radio imaging of this galaxy at $1.4 \mathrm{GHz}$ has been presented by Condon et al. (1990) and at $8.4 \mathrm{GHz}$ by C91. CO observations of VV 114 were reported by Yun, Scoville, \& Knop (1994), while submillimeter continuum imaging of this system is presented in Frayer et al. (1999).

The images presented in Knop et al. (1994), ranging in wavelengths from $B(0.44 \mu \mathrm{m})$ to $L(3.5 \mu \mathrm{m})$, show the core of the eastern galaxy is nearly invisible at $B$ and becomes brighter with respect to the western nucleus at longer wavelengths. In the longest wavelength images of Knop et al. the eastern core is clearly double, with a separation between peaks of $\sim 2^{\prime \prime}(800 \mathrm{pc})$. In the eastern system the southwestern component becomes increasingly dominant at longer wavelengths. In the $L$-band image of Knop et al. the southwestern component is the only one detected. Infrared Space Observatory (ISO) imaging of this galaxy at $7 \mu \mathrm{m}$ (V. Charmandaris 2000, private communication) shows a bright peak apparently associated with the eastern nucleus with an extension to the west.

The only portion of the galaxy detected in the MIRLIN and LWS imaging was the eastern source. The $12.5 \mu \mathrm{m}$ image of this nucleus is presented in Figure $1 a$, along with the $3.2 \mu \mathrm{m}$ image obtained with NIRC, the $2.2 \mu \mathrm{m}$ NICMOS image from S00, and the $8.4 \mathrm{GHz}$ image of $\mathrm{C} 91$. The $12.5 \mu \mathrm{m}$ image presented in Figure $1 a$ is smoothed with a Gaussian with half the diffraction limited FWHM to improve the signal-to-noise ratio $(\mathrm{S} / \mathrm{N})$. The effective resolution of this image is 0 ". 34 , i.e., $10 \%$ greater than the formal diffraction limit at $12.5 \mu \mathrm{m}$.

The two peaks in the eastern nucleus are clearly seen at $12.5 \mu \mathrm{m}$; both components are resolved in the image. In the subsequent discussion, they are referred to as $\mathrm{VV} 114 \mathrm{E}_{\mathrm{NE}}$ and VV $114 \mathrm{E}_{\mathrm{SW}}$, respectively. VV $114 \mathrm{E}_{\mathrm{NE}}$ is centrally concentrated with an apparent size of 0.44 . The VV $114 \mathrm{E}_{\mathrm{SW}}$ component is complex, with a main northern component having an apparent size of 0.51 and several sources to the south, separated from the brighter peak by $0.6-0.8$. The peak positions of VV $114 \mathrm{E}_{\mathrm{NE}}$ and $\mathrm{VV} 114 \mathrm{E}_{\mathrm{Sw}}$, as determined in the NICMOS $2.2 \mu \mathrm{m}$ image, are marked in all four images of Figure 1a. The marks are located at the same relative positions in all four panels of the montage.
The $3.2 \mu \mathrm{m}$, image, with lower angular resolution, shows structure that appears quite similar to that at $12.5 \mu \mathrm{m}$. At $3.2 \mu \mathrm{m}$, VV $114 \mathrm{E}_{\mathrm{Sw}}$ is only marginally resolved, while VV $114 \mathrm{E}_{\mathrm{NE}}$ is approximately 0.7 in diameter and apparently at the same orientation as the source seen at $2.2 \mu \mathrm{m}$. At $3.2 \mu \mathrm{m}$ VV $114 \mathrm{E}_{\mathrm{Sw}}$ appears displaced from the corresponding 12.5 $\mu \mathrm{m}$ source by 0.1 . The radio image shown in Figure $1 a$ shows a general similarity to the $12.5 \mu \mathrm{m}$ image, with a bright isolated northeast component and a southwest source that consists of several distinct components separated by $\sim 0.4-0 " .8$. The radio map of VV $114 \mathrm{E}_{\mathrm{Sw}}$ shows that the components are reasonably close in peak brightness, like the $12.5 \mu \mathrm{m}$ image.

At $2.2 \mu \mathrm{m}$, the structure of VV 114E is significantly different from the mid-infrared and radio structure. VV $114 \mathrm{E}_{\mathrm{NE}}$ shows a significant ellipticity at $2.2 \mu \mathrm{m}$, with a FWHM size of $0.8 \times 00^{\prime \prime} 4$ at a position angle of $60^{\circ}$, suggestive of a stellar nucleus. VV $114 \mathrm{E}_{\mathrm{Sw}}$ breaks up into two sources, a bright, apparently unresolved source nearly coincident in position with the $12.5 \mu \mathrm{m}$ source, and a fainter source 0 " 86 to the southwest of the brighter pointlike source.

While consistent in overall structure, the images do not agree in detail at the different wavelengths. The registration between images at different wavelengths is based on the assumption that $\mathrm{VV} 114 \mathrm{E}_{\mathrm{NE}}$ is spatially coincident at all wavelengths. Since this source is reasonably compact and isolated from other sources by $\sim 2^{\prime \prime}$ at all wavelengths, we believe that this is a reasonable assumption.

At 2.2 and $3.2 \mu \mathrm{m}$, the positions of $\mathrm{VV} 114 \mathrm{E}_{\mathrm{NE}}$ and $\mathrm{VV}$ $114 \mathrm{E}_{\mathrm{Sw}}$ agree in position angle to less than $1^{\circ}$ and in separation to within $0^{\prime \prime} 03$. In addition, as noted above, at $3.2 \mu \mathrm{m}$, the northeastern source appears slightly elongated in the same sense as the elliptical shape of this source at $2.2 \mu \mathrm{m}$. There is also a slight distortion of the lower level contours of the southwestern source at $3.2 \mu \mathrm{m}$, consistent with a fainter source at the location of the faint source to the southwest of the point source in the $2.2 \mu \mathrm{m}$ image. Thus given the angular resolution and $\mathrm{S} / \mathrm{N}$ in the $3.2 \mu \mathrm{m}$ image, these images appear the same.

The 12.5 and $2.2 \mu \mathrm{m}$ images differ in detail. In both cases, the northeastern source is extended, and there is some suggestion of extended emission at $12.5 \mu \mathrm{m}$ at a similar position angle as the major axis of the $2.2 \mu \mathrm{m}$ image. There is a significant disagreement in position and morphology of the southwestern source between these wavelengths. This is illustrated in Figure $1 b$, where the $12.5 \mu \mathrm{m}$ contours are overlaid on a gray-scale version of the $2.2 \mu \mathrm{m}$ image. The northeast and southwest peaks in the two images appear at the same position angle of $80^{\circ}$, but the location of the brightest component of the southwestern source disagrees by 0 ." 1 between the two wavelengths. Furthermore, the secondary peaks to the south and southwest of the southwestern peak at $12.5 \mu \mathrm{m}$ do not appear to have any corresponding peaks in the $2.2 \mu \mathrm{m}$ image, while the faint peak to the southwest of the bright $2.2 \mu \mathrm{m}$ peak has no counterpart in the $12.5 \mu \mathrm{m}$ image.

The largest discrepancies in location of the peaks are between the $12.5 \mu \mathrm{m}$ image and the $8.4 \mathrm{GHz}$ image. As can be seen in Figure 1a, the separations between the VV $114 \mathrm{E}_{\mathrm{NE}}$ and VV $114 \mathrm{E}_{\mathrm{Sw}}$ peaks differ by 0.3 or $100 \mathrm{pc}$. There is no a priori means of knowing whether the registration we have chosen (forcing VV $114 \mathrm{E}_{\mathrm{NE}}$ to coincide at both wavelengths) is valid, or whether forcing the peak of VV $114 \mathrm{E}_{\mathrm{SW}}$ to coincide might be appropriate. In either case, a 


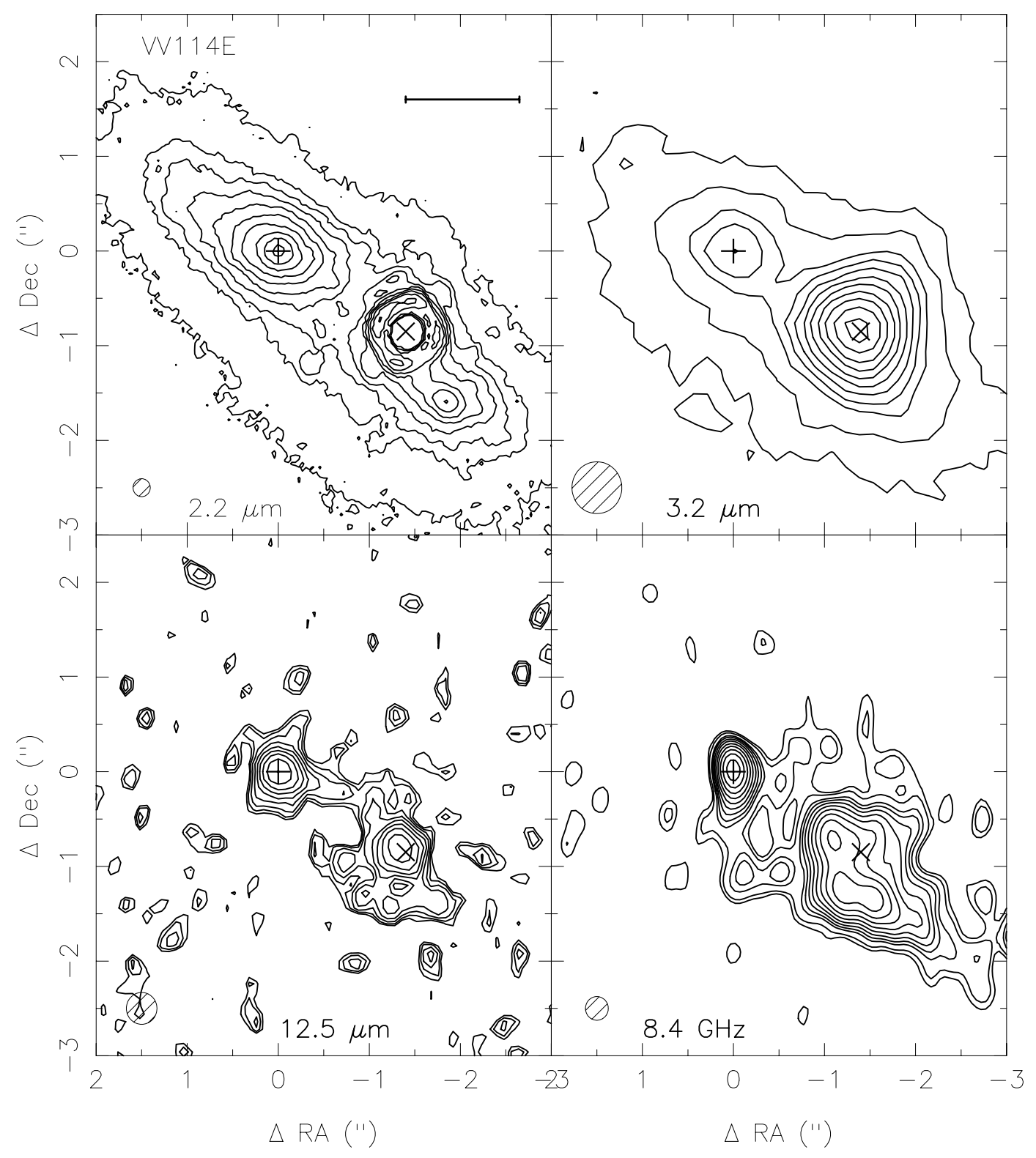

FIG. $1 a$

Fig. 1.-(a) Montage of contour plots of VV $114 \mathrm{E}$ at $2.2 \mu \mathrm{m}, 3.2 \mu \mathrm{m}, 12.5 \mu \mathrm{m}$, and $8.4 \mathrm{GHz}$. In this and all subsequent figures, north is up and east to the left, and the FWHM size of the point-spread function is shown separately in the panel for each wavelength as the hatched circle. In this and all subsequent figures, contour levels are spaced by a factor of 1.34 . The first contour is set to $90 \%$ of the peak brightness so that the third contour down represents $50 \%$ of the peak brightness. In this and all subsequent figures, unless otherwise indicated, the $2.2 \mu \mathrm{m}$ images are from S00, while the radio images are from C91. In this and all subsequent figures that display contour plots of the galaxies, the horizontal bar in one panel represents $500 \mathrm{pc}$ at the galaxy. In $(a)$ the origin is chosen to be at the location of the northeast peak in the $2.2 \mu \mathrm{m}$ image. The astrometric registration of the images at the different wavelengths is discussed in the text. The locations of the brightest peaks in the $2.2 \mu \mathrm{m}$ image are indicated by a plus sign and times cross in the same location in each panel. (b) Contour map of VV 114E at $12.5 \mu \mathrm{m}$ overlaid on the corresponding gray-scale image of $8.4 \mathrm{GHz}$ emission (left). Center: Overlay of the $3.2 \mu \mathrm{m}$ emission contours on the corresponding gray-scale image of $8.4 \mathrm{GHz}$ emission. Right: Overlay of the $12.5 \mu \mathrm{m}$ emission contours on the corresponding gray-scale image of the 2.2 $\mu \mathrm{m}$ emission. In these and all subsequent gray-scale images, the wavelength of the contoured image is given in the lower left corner and that of the gray-scale image in the lower right. (c) Contour map of the full VV 114 field at $3.2 \mu \mathrm{m}$ overlaid on a gray-scale image of the same region at $2.2 \mu \mathrm{m}$. (d) Spectral energy distributions for $(t o p)$ all of VV 114, VV 114E, VV 114 $\mathrm{E}_{\mathrm{NE}}$, and VV 114. $\mathrm{E}_{\mathrm{SW}}$ and (bottom) all of VV 114 repeated and VV 114W. The data are plotted as flux per octave, $v f_{v}$ vs. wavelength. In the top panel, the integrated fluxes, taken from the IRAS and 2MASS data, are shown as filled circles, along with photometry in a 4" diameter beam centered on VV 114E ( filled squares). In addition, photometry in 1" diameter beams centered on VV 114 $\mathrm{E}_{\mathrm{SW}}$ (times cross) and VV $114 \mathrm{E}_{\mathrm{NE}}$ (plus sign) as indicated in $(a)$ is also shown (open squares). In this and all subsequent SEDs, the beam diameter for the photometry is indicated next to the identifying symbol. In this and the subsequent SEDs, the photometric points made with the same beam size are joined together with straight lines intended only to guide the eye. They are not intended to indicate the absence of significant structure between the data points presented, e.g., due to PAH emission or silicate absorption. The mid-infrared photometry is from data presented here. The $2.2 \mu \mathrm{m}$ data are from the NICMOS imaging of S00, while the $3.2 \mu \mathrm{m}$ data are from NIRC. In the bottom panel the integrated fluxes are repeated, as well as the measured emission for VV $114 \mathrm{~W}$ in a $4^{\prime \prime}$ diameter beam centered on the western peak shown in $(c)$. 


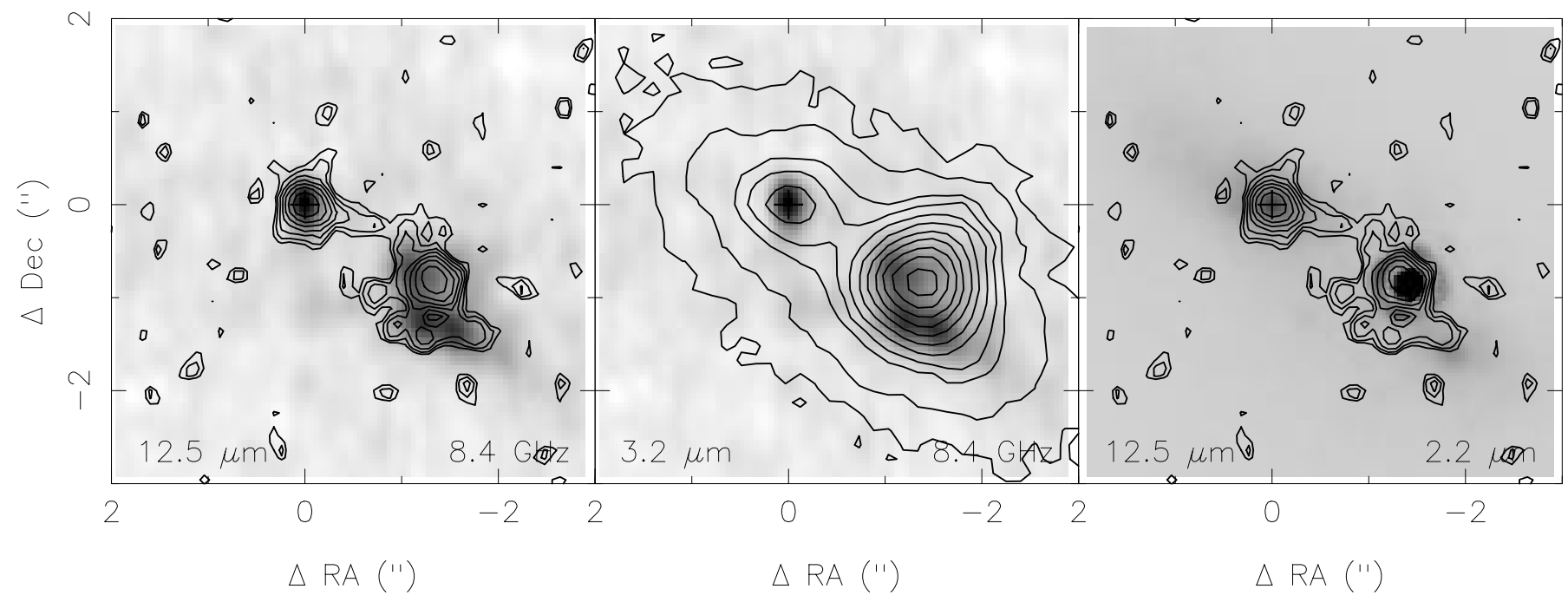

FIG. $1 b$

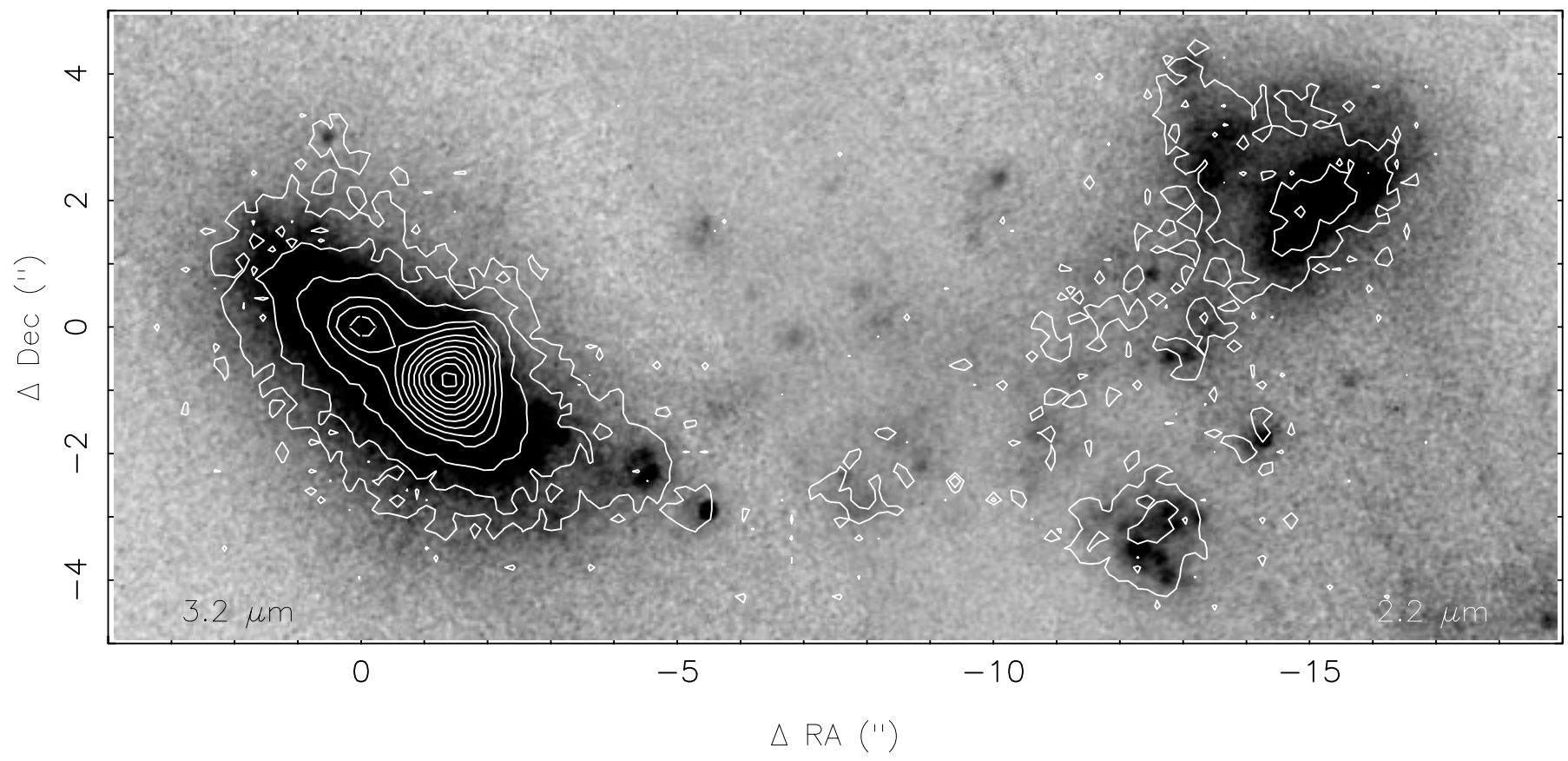

FIG. 1c

discrepancy exists between the locations of the mid-infrared and radio peaks.

Figure $1 b$ also shows the 12.5 and the $3.2 \mu \mathrm{m}$ contours overlaid separately on a gray-scale version of the $8.4 \mathrm{GHz}$ image. In both cases, the location of the peak of the southwestern source in the "thermal" infrared does not coincide with the radio peak. As in the case of comparing the sources at 12.5 and $2.2 \mu \mathrm{m}$, the northeast and southwest peaks fall along the same position angle, but the separations disagree. The separation of the radio peaks is 1 ."35, compared with 1.55 in the thermal infrared. The structure to the south of the southwestern peak appears to agree much better between the radio and mid-infrared images, showing agreement in both location and elongation.

In summary, assuming VV $114 \mathrm{E}_{\mathrm{NE}}$ is spatially coincident at all wavelengths, the VV $114 \mathrm{E}_{\mathrm{Sw}}$ peaks coincide at 2.2 and $3.2 \mu \mathrm{m}$ and are displaced from the $12.5 \mu \mathrm{m}$ peak by 0.1 ( 30 pc). The $12.5 \mu \mathrm{m}$ peak is displaced from the $8.4 \mathrm{GHz}$ peak by $0.2(80 \mathrm{pc})$; at $12.5 \mu \mathrm{m} \mathrm{VV} 114 \mathrm{E}_{\mathrm{SW}}$ lies between the 8.4 $\mathrm{GHz}$ and $2.2 \mu \mathrm{m}$ peak, and they all lie on the same line from VV $114 \mathrm{E}_{\mathrm{NE}}$.

The flux density of VV 114 measured by $I R A S$ at $12 \mu \mathrm{m}$ is $0.8 \mathrm{Jy}$ in an unresolved beam and $1.1 \mathrm{Jy}$ in total. The flux density measured in the imaging of VV $114 \mathrm{E}$ is $0.34 \mathrm{Jy}$ at $12.5 \mu \mathrm{m}$, significantly less than the IRAS values. To attempt to detect additional emission, the position of peak extended infrared emission in VV $114 \mathrm{~W}$ based on the ISO image of Laurent et al. (2000) was imaged as well. This location is 14" west of the VV 114E position. No flux was detected in the Keck image at $12.5 \mu \mathrm{m}$, with a limit of $8 \pm 5 \mathrm{mJy}$ in a $4^{\prime \prime}$ diameter beam. For this imaging, the chopping amplitude was $10^{\prime \prime}$. The nondetection of the western source places an upper limit of $\sim 1 \mathrm{mJy} \operatorname{arcsec}^{-2}$ on the surface brightness of the emission at this location. 


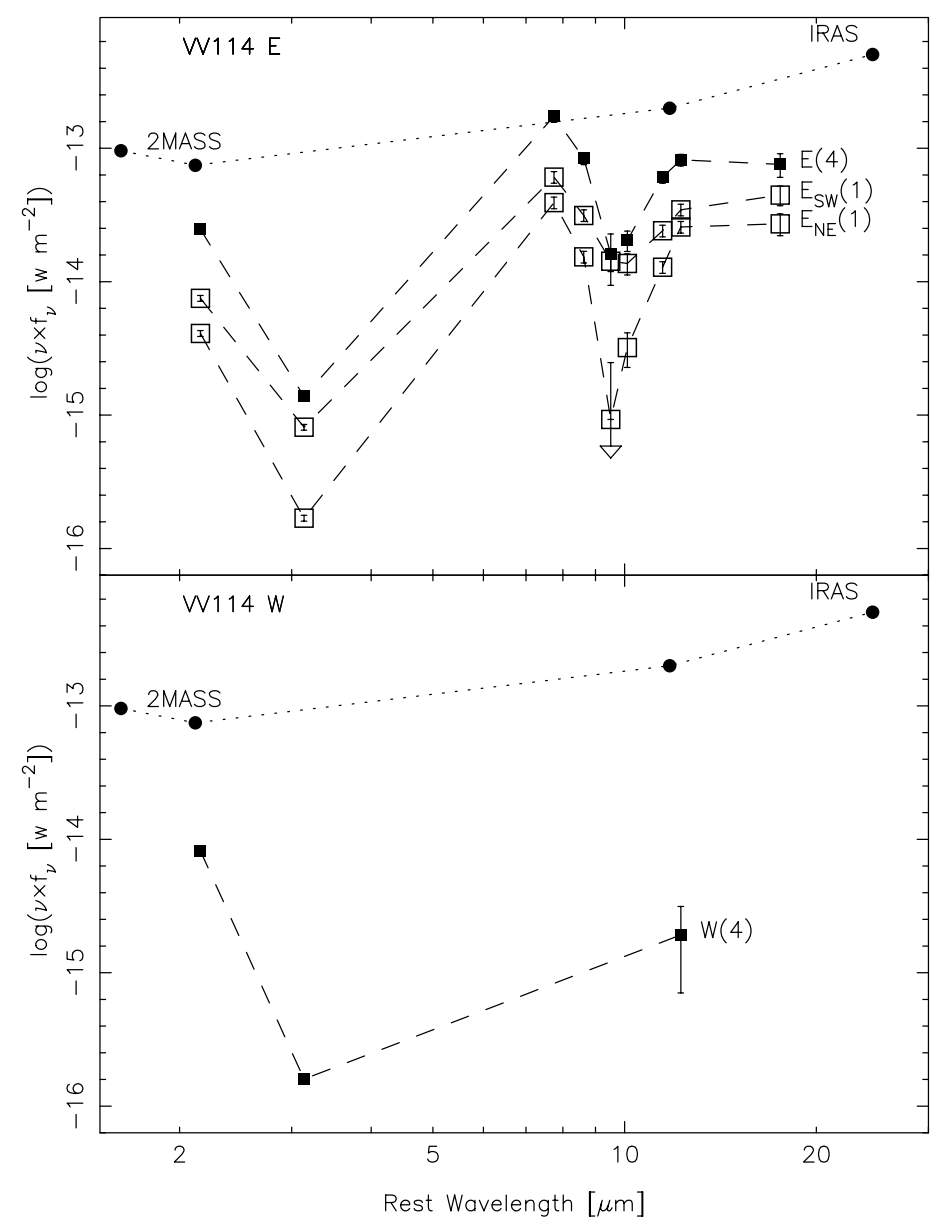

Fig. 1d

The western galaxy in the VV 114 pair is detected at 3.2 $\mu \mathrm{m}$ and is shown in Figure 1c. The full field containing both systems is shown at 2.2 and $3.2 \mu \mathrm{m}$, where the $3.2 \mu \mathrm{m}$ contours are overlaid on the $2.2 \mu \mathrm{m}$ gray scale. The $3.2 \mu \mathrm{m}$ NIRC image shows that there is low surface brightness, extended emission associated with the western galaxy, and the interaction region between the two galaxies. The $3.2 \mu \mathrm{m}$ emission is comprised of a combination of photospheric, nebular, and dust emission. The $3.2 \mu \mathrm{m}$ filter includes the $3.3 \mu \mathrm{m}$ feature commonly identified as due to polycyclic aromatic hydrocarbons (PAHs; e.g., Dale et al. 2001), and so there is a contribution from this mechanism as well. The detection of extended emission at $3.2 \mu \mathrm{m}$, in addition to the detection of substantial extended emission in the IRAS photometry with a $\sim 1^{\prime}$ beam, argues that the nondetection of the majority of the IRAS $12 \mu \mathrm{m}$ flux is probably the result of the difficulty in detecting low surface brightness extended emission in the thermal infrared.

The spectral energy distributions for the sources in VV 114 from 1.2 to $25 \mu \mathrm{m}$ are presented in Figure $1 d$ as flux per octave $\left(v f_{v}\right)$. In addition to the total fluxes from IRAS and 2MASS, photometry in a 4 " diameter beam centered on VV $114 \mathrm{E}$, as well as in $1^{\prime \prime}$ diameter beams centered on VV $114 \mathrm{E}_{\mathrm{NE}}$ and VV $114 \mathrm{E}_{\mathrm{Sw}}$, are presented to illustrate how the SEDs change with observing beam. The small-beam photometry at $2.2 \mu \mathrm{m}$ was scaled from the NICMOS image in S00, while the $3.2 \mu \mathrm{m}$ data are from our NIRC imaging. The bottom panel illustrates the photometry at the corresponding location in VV $114 \mathrm{~W}$.

Both VV $114 \mathrm{E}_{\mathrm{NE}}$ and VV $114 \mathrm{E}_{\mathrm{Sw}}$ show a significant drop in flux at $10 \mu \mathrm{m}$ as compared with the observed flux at 8 and $12 \mu \mathrm{m}$. This is generally attributed to absorption by cold silicate dust overlaying warmer emission. A substantial uncertainty in quantifying the overlaying absorption arises from the contribution to the underlying emission from the aromatic (PAH) features. The spectrum of VV 114 reported by Dudley (1999) with a 5.5 diameter beam includes all of the emission seen in the image in Figure 1a. This spectrum shows an emission feature at $11.3 \mu \mathrm{m}$ (rest wavelength) attributed to PAH emission. The spectrum of Dudley, if smoothed to the resolution of the filters used for our imaging, is consistent with the apparent ratio of fluxes at 11.7 and $12.5 \mu \mathrm{m}$ as shown for the flux from VV $114 \mathrm{E}$ in the large beam shown in Figure $1 d$.

The separate spectral energy distributions (SEDs) of VV $114 \mathrm{E}_{\mathrm{NE}}$ and VV $114 \mathrm{E}_{\mathrm{Sw}}$ differ significantly. The decrease in flux density at $10 \mu \mathrm{m}$ of VV $114 \mathrm{E}_{\mathrm{NE}}$ is substantially deeper than in VV $114 \mathrm{E}_{\mathrm{Sw}}$. The composite mid-infrared spectrum of galaxies presented by Dale et al. (2001), if representative of the underlying emission spectrum for VV 114E, suggests that the extinction of an intrinsic PAH-like spectrum would lead to an absorption optical depth of about 0.5 at $10 \mu \mathrm{m}$ in VV $114 \mathrm{E}_{\mathrm{Sw}}$. This is also consistent with the fact that the southwest source appears to dominate the VV 114E flux 
throughout most of the $10 \mu \mathrm{m}$ wavelength range. VV $114 \mathrm{E}_{\mathrm{NE}}$ appears to have an optical depth $\tau$ in the range 1-2 greater than that of $\mathrm{VV} 114 \mathrm{E}_{\mathrm{Sw}}$ at $9.7 \mu \mathrm{m}$ (i.e., a net differential optical depth $\tau$ of $1.5-2.5$ at $\sim 10 \mu \mathrm{m}$ ). The absorption optical depths cited here are by comparison with zero absorption at 8 or $12 \mu \mathrm{m}$.

Yet another complication is the fact that the dust optical depth is not zero at 8 and $12 \mu \mathrm{m}$, as assumed above. Li \& Draine (2001) have recently compiled the properties of interstellar dust opacity (predominantly due to silicate absorption) that would result in an increase of a factor of 1.3 to the optical depths derived above to take into account the extinction at 8 and $12 \mu \mathrm{m}$.

Thus we infer that the silicate optical depths are $\sim 0.7$ and 2-3 for VV $114 \mathrm{E}_{\mathrm{SW}}$ and VV $114 \mathrm{E}_{\mathrm{NE}}$, respectively. These results, which imply that VV $114 \mathrm{E}_{\mathrm{NE}}$ is more heavily obscured than VV $114 \mathrm{E}_{\mathrm{sw}}$, contradict the extinctions derived from the near-infrared imaging of these sources. As can be seen directly from Figure 1 of Knop et al. (1994), VV $114 \mathrm{E}_{\mathrm{SW}}$ is significantly "redder" than VV $114_{\mathrm{NE}}$; i.e., it becomes significantly brighter than VV $114_{\mathrm{NE}}$ at $2.2 \mu \mathrm{m}$ compared with $0.8 \mu \mathrm{m}$. To the extent that this reflects over- laying extinction, it suggests substantially more extinction obscuring VV $114 \mathrm{E}_{\mathrm{SW}}$ than VV $114 \mathrm{E}_{\mathrm{NE}}$.

\section{2. $N G C 1614$ (= Arp 186)}

NGC 1614 is a strongly interacting galaxy at a redshift of

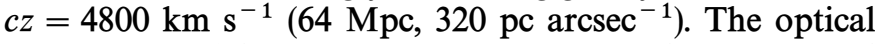
photograph of Arp (1966) shows crossed tails with a total extent of $\sim 30 \mathrm{kpc}$. Its infrared luminosity is $4 \times 10^{11} L_{\odot}$ (Soifer et al. 1987). A detailed visible, infrared, and radio study by Neff et al. (1990) shows an H II optical spectrum and tidal tails. The radio image at $5 \mathrm{GHz}$ shows a ring $\sim 1$ 1".2 (380 pc) in diameter (Neff et al.). High-resolution near-infrared imaging and spectroscopy (Alonso-Herrero et al. 2001) shows a starburst nucleus of $\sim 0.3(100 \mathrm{pc})$ diameter revealed through strong photospheric $\mathrm{CO}$ absorption in supergiants. This nucleus is surrounded by a ring of very large $\mathrm{H}$ II regions. The ring diameter of $\sim 1.2(380 \mathrm{pc})$ is traced in the $\mathrm{P} \alpha$ image (Alonso-Herrero et al.). Miles et al. (1996) have reported imaging of NGC 1614 at $11.7 \mu \mathrm{m}$ with 1 " resolution.

Figure $2 a$ shows the $12.5 \mu \mathrm{m}$ contour map of NGC 1614, the $4.8 \mathrm{GHz}$ radio contours of Neff et al. (1990) and the 2.2

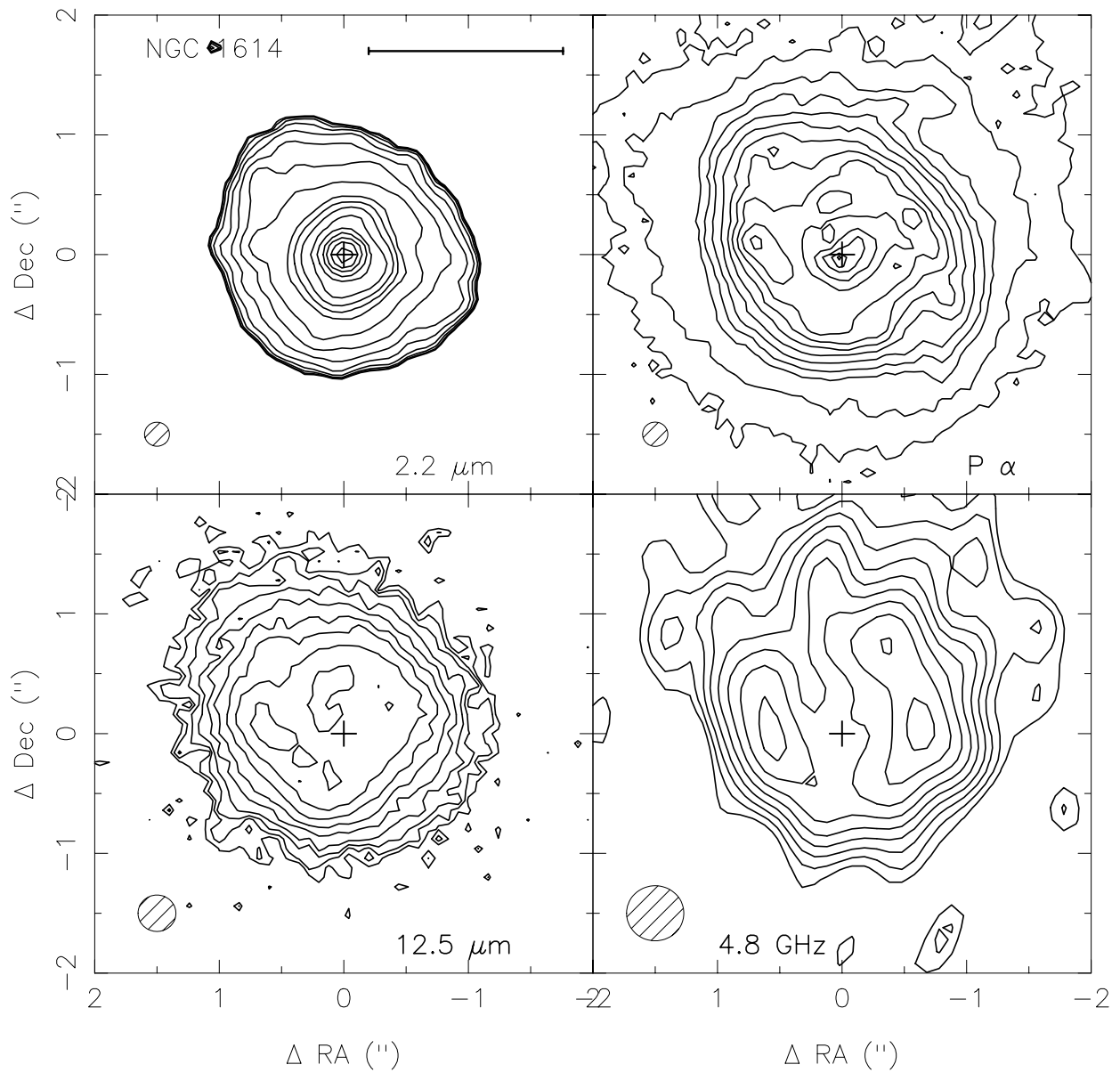

FIG. $2 a$

FIG. 2.-(a) Montage of contour plots of NGC 1614 at $\mathrm{P} \alpha(1.87 \mu \mathrm{m}), 2.2 \mu \mathrm{m}, 12.5 \mu \mathrm{m}$, and $4.8 \mathrm{GHz}$ is presented. The origin is chosen to be at the peak emission in the $2.2 \mu \mathrm{m}$ image. The astrometric registration of the images at the different wavelengths is discussed in the text. The location of the center in each image is indicated by a plus sign in each panel. The $2.2 \mu \mathrm{m}$ broadband and the $1.87 \mu \mathrm{m} \mathrm{P} \alpha$ NICMOS images are from Alonso-Herrero et al. (2001). (b) Contour map of NGC 1614 at $12.5 \mu \mathrm{m}$ overlaid on the corresponding gray-scale image of P $\alpha$ emission (left). An overlay of the $12.5 \mu \mathrm{m}$ emission contours on the corresponding gray-scale image of $4.8 \mathrm{GHz}$ emission is presented in the right panel. (c) Spectral energy distribution of NGC 1614. The filled circles represent the integrated fluxes taken from IRAS and 2MASS data. The aperture photometry in a 4 " diameter beam is represented by filled squares, the photometry in a $2^{\prime \prime}$ diameter beam is represented by open circles. The $2^{\prime \prime}$ diameter was set to include the ring of emission seen in $(b)$. The $2.2 \mu \mathrm{m}$ photometric data are from the NICMOS image on NGC 1614 (Alonso-Herrero et al. 2001) and Carico et al. (1988). 


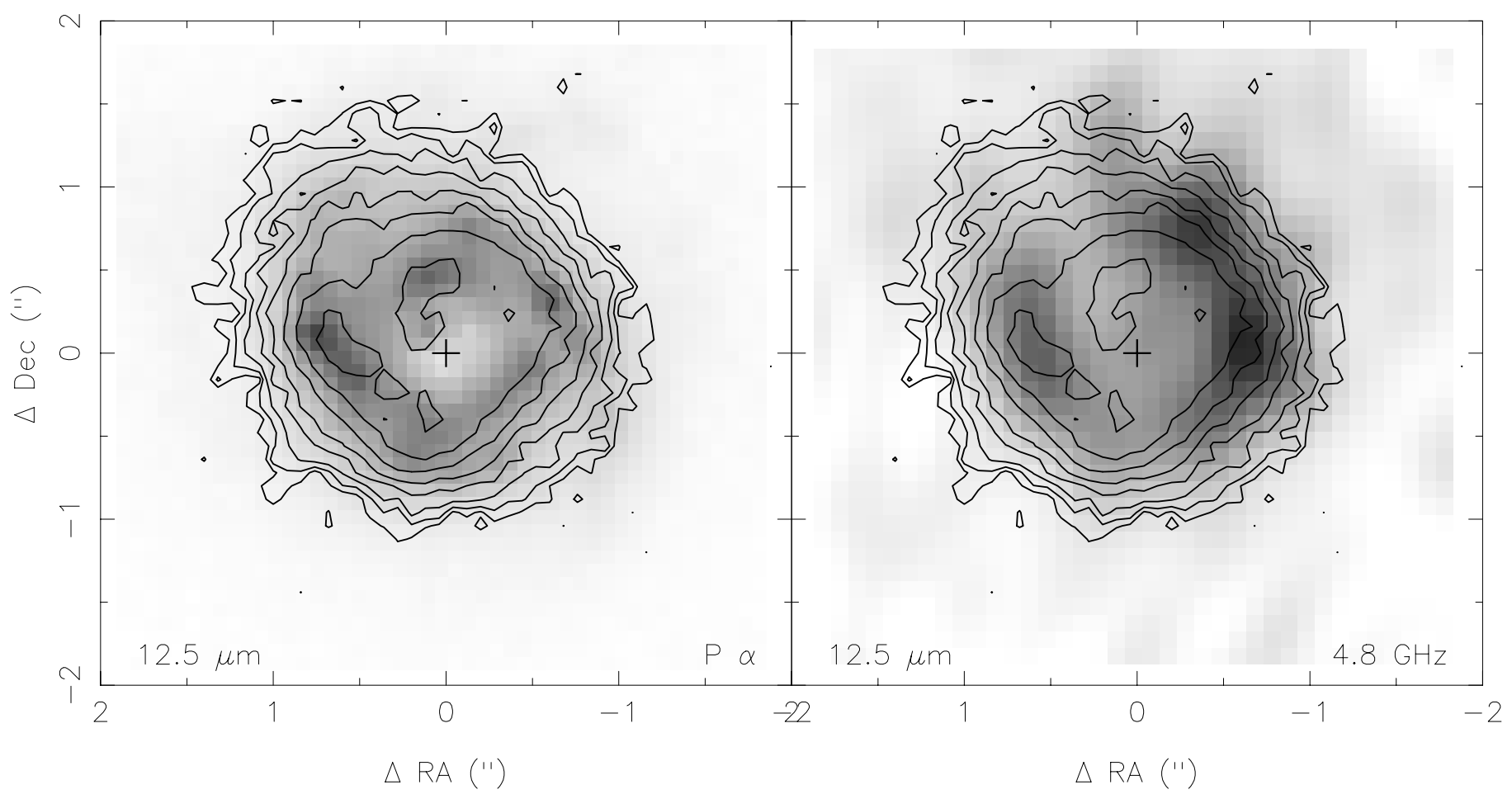

FIG. $2 b$

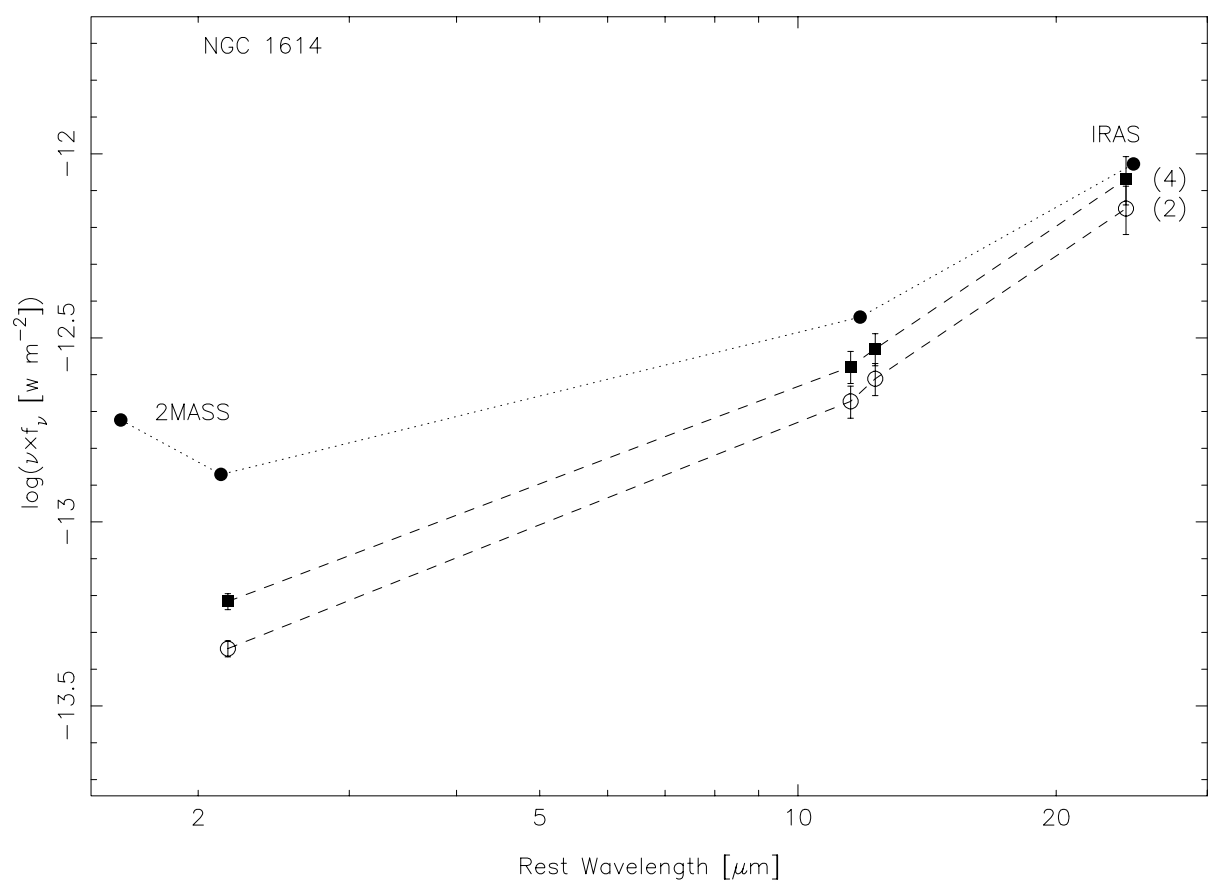

FIG. $2 c$

$\mu \mathrm{m}$ broadband and $1.87 \mu \mathrm{m} \mathrm{P} \alpha$ NICMOS images from Alonso-Herrero et al. (2001). The $12.5 \mu \mathrm{m}$ image, obtained in excellent (diffraction-limited) 0"30 seeing, shows a ringlike structure of diameter $\sim 1$ ".2 and overall extent of $\sim 1$ ".7 that appears virtually identical in overall appearance to the ring seen in the radio at $4.8 \mathrm{GHz}$ and in $\mathrm{P} \alpha$.

A detailed comparison of the $12.5 \mu \mathrm{m}$ and the $\mathrm{P} \alpha$ and 4.8 $\mathrm{GHz}$ images is presented in Figure 2b, where the contours of the mid-infrared image are overlaid on the gray-scale images of $\mathrm{P} \alpha$ and $4.8 \mathrm{GHz}$ radio emission. The images were superposed by matching by eye the centroids at the different wavelengths. The peaks in the thermal infrared, $P \alpha$, and 4.8 $\mathrm{GHz}$ images are very well matched, particularly to the southeast where the peaks agree in size and orientation.

In contrast to the mid-infrared and P $\alpha$ images, the $2.2 \mu \mathrm{m}$ continuum image (Fig. 2a) does not reveal a ringlike structure. Rather the $2.2 \mu \mathrm{m}$ continuum shows a strong central peak, with an overall size similar to the size of the midinfrared image. This results from the fact that the $2.2 \mu \mathrm{m}$ light traces the stars that peak at the nucleus, while the 
mid-infrared, radio, and $\mathrm{P} \alpha$ images trace the ring of current star formation.

Figure $2 c$ shows the SED of NGC 1614, comparing the photometric measurements obtained with the Keck images in $2^{\prime \prime}$ and $4^{\prime \prime}$ diameter beams and the IRAS observations. This plot shows that the Keck observations detect $\sim 87 \%$ of the flux measured at $12 \mu \mathrm{m}$ with $I R A S$ in a $4^{\prime \prime}$ beam and $72 \%$ of the IRAS $12 \mu \mathrm{m}$ flux in a $2^{\prime \prime}$ beam. The photometry shows that the mid-infrared emission is predominantly confined to a nuclear region $\sim 1^{\prime \prime} .7(550 \mathrm{pc})$ in diameter (FWHM) with less than $30 \%$ of the emission extending outside this diameter. The mid-infrared spectrum presented in Roche et al. (1991) shows a suggestion of both PAH emission and silicate absorption. We do not have adequate photometric data to distinguish $\mathrm{PAH}$ emission from silicate absorption. The images at 11.7 and $12.5 \mu \mathrm{m}$ show similar structure, showing that grossly there are not significantly different spectra in different locations in the starburst region.

\section{3. $N G C 2623$ (= Arp 243)}

NGC 2623 (=Arp 243) is a strongly interacting galaxy at a redshift of $c z=5535 \mathrm{~km} \mathrm{~s}^{-1}\left(74 \mathrm{Mpc}, 370 \mathrm{pc} \operatorname{arcsec}^{-1}\right)$ with an infrared luminosity of $3 \times 10^{11} L_{\odot}$ (Soifer et al. 1987). The optical image (Arp 1966) shows two opposing tidal tails extending over $120^{\prime \prime}(45 \mathrm{kpc})$. Near-infrared NICMOS imaging ( $\mathrm{S} 00$ ) shows a single bright nucleus and surrounding galaxy, and the $8.4 \mathrm{GHz}$ image (C91) shows a similar structure of a bright compact core with an east-west elongated structure.

The $12.5 \mu \mathrm{m}$ image of NGC 2623 is shown in Figure $3 a$, along with the $8.4 \mathrm{GHz}$ and NICMOS $2.2 \mu \mathrm{m}$ images. The angular resolution of the $12.5 \mu \mathrm{m}$ image is 0 ".7, significantly lower resolution than either the $8.4 \mathrm{GHz}(0.3)$ or $2.2 \mu \mathrm{m}$ $(0$ ".22) images. The $12.5 \mu \mathrm{m}$ image has been registered with respect to the radio and $2.2 \mu \mathrm{m}$ images by assuming that the peak brightness at $12.5 \mu \mathrm{m}$ agrees with the peaks at the other wavelengths.

As can be seen from this figure, the structure of the 12.5 $\mu \mathrm{m}, 2.2 \mu \mathrm{m}$, and $8.4 \mathrm{GHz}$ images are all very similar and show similar sizes. The observed FWHM size of the $12.5 \mu \mathrm{m}$ source is $1^{\prime \prime} .0 \times 00^{\prime \prime} .7$ or an intrinsic size of 0 ". $7 \times<0$."35 (260 pc $\times<130 \mathrm{pc}$ ), with the major axis oriented east-west. The deconvolved size of the radio source is $0.43 \times 0$ ".29 (160 pc $\times 110 \mathrm{pc})$. The overall extent of the $12.5 \mu \mathrm{m}$ and $8.4 \mathrm{GHz}$ emission agrees quite well, being $\sim 2^{\prime \prime} \times 1^{\prime \prime} .5(740 \times 550 \mathrm{pc})$ with the major axis oriented in the east-west direction. The excellent agreement between the $12.5 \mu \mathrm{m}$ and $8.4 \mathrm{GHz}$ maps of NGC 2623 is illustrated directly in Figure $3 b$, where the $12.5 \mu \mathrm{m}$ contours are overlaid on the gray-scale image of the $8.4 \mathrm{GHz}$ image. The $2.2 \mu \mathrm{m}$ NICMOS image shows a circular core with a size of $0.2(75 \mathrm{pc})$ and a larger nuclear region having the same size and orientation as that seen at $12.5 \mu \mathrm{m}$ and $8.4 \mathrm{GHz}$.

The flux densities presented in Table 2 and fluxes shown in Figure $3 c$ show that the $12.5 \mu$ m flux density measured in a 4 " diameter beam represents $80 \%$ of the total flux density measured by $I R A S$ at $12 \mu \mathrm{m}$, while $65 \%$ of the $I R A S$ flux density is contained in a $1^{\prime \prime}$ diameter beam. As in NGC 1614 , the vast majority of the mid-infrared emission in NGC 2623 is confined to the nuclear region with a size of less than $400 \mathrm{pc}$. The $8-13 \mu \mathrm{m}$ spectrum presented by Dudley (1999) is consistent with the observations reported here and shows a PAH emission feature affecting the 11.7 $\mu$ m flux measurement. The mid-infrared spectrum suggests significant silicate absorption of an underlying PAH emission spectrum, although quantifying this is highly uncertain.

$$
\text { 4.4. } N G C 3690+I C 694 \text { (=Arp 299=Mrk 171) }
$$

NGC $3690+$ IC 694 (= Arp 299, Mrk 171) is one of the most extensively studied interacting starburst galaxies known. Its optical morphology (Arp 1966) shows two main bodies with many bright internal knots and diffuse tidal debris extending over $90^{\prime \prime}(20 \mathrm{kpc})$. This system was first shown to be a bright infrared and radio source by Gehrz, Sramek, \& Weedman (1983) who showed $10 \mu \mathrm{m}$ emission extending over nearly $40^{\prime \prime}(8 \mathrm{kpc})$, encompassing both galactic nuclei. The redshift of NGC 3690 is $c z=3120 \mathrm{~km} \mathrm{~s}^{-1}$ (41 Mpc, $\left.210 \mathrm{pc} \operatorname{arcsec}^{-1}\right)$. IRAS measurements showed a total bolometric luminosity of $8 \times 10^{11} L_{\odot}$, making this the most luminous galaxy in this study (Soifer et al. 1987) and very close to the ultraluminous galaxy limit (Sanders et al. 1988).

The optical spectrum is classified as coming from a $\mathrm{H} \mathrm{II}$ region (e.g., Villeux et al. 1995), while the mid-infrared spectrum of Dudley (1999) shows PAH emission and silicate absorption. Sargent \& Scoville (1991) showed this system to be rich in molecular gas, while extensive near-infrared studies have been reported by Sugai et al. (1999) and Satyapal et al. (1999) among others. A detailed near-infrared study of NGC 3690 with NICMOS on HST has been reported by Alonso-Herrero et al. (2000). Previous groundbased mid-infrared imaging of NGC 3690 has been reported by Miles et al. (1996) and Keto et al. (1997).

Figures $4 a$ and $4 b$ show the $12.5 \mu \mathrm{m}$ image along with the

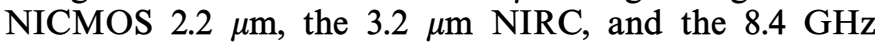
images of NGC 3690/IC 694. The five nuclei identified at 2.2 $\mu \mathrm{m}$ are marked; the marks are reproduced at the same relative positions in all four panels of Figures $4 a$ and $4 b$. We use here the nomenclature for the sources introduced by Gehrz, Sramek, \& Weedman (1983) and Wynn-Williams et al. (1991) of A, B1, B2, C, and C'. Figure $4 a$ shows the entire extent of the emission, while Figure $4 b$ shows detailed maps of the western sources. The astrometric registration of the maps at different wavelengths is based on morphological similarities and direct measurements. The $8.4 \mathrm{GHz}$ image of C91 and the $2.2 \mu \mathrm{m}$ image of Alonso-Herrero et al. (2000) were overlaid by assuming spatial coincidence of sources A and $\mathrm{B} 1$. The 2.2 and $3.2 \mu \mathrm{m}$ images agree spatially to within 0 . 05 , based on NIRC measurements. After superposing the $12.5 \mu \mathrm{m}$ image of source B1, at $12.5 \mu \mathrm{m}$ the western sources (B1, B2, C, and $\mathrm{C}^{\prime}$ ) match the locations at $3.2 \mu \mathrm{m}$ to less than 0.05 . Because of the extent of NGC 3690, the nod amplitude was increased to 20", and all four sources of NGC $3690 \mathrm{~W}$ were thus imaged on one frame at $12.5 \mu \mathrm{m}$ and thus were registered accurately with respect to each other, as seen in Figure $4 b$. NGC $3690 \mathrm{~A}$ was imaged separately at $12.5 \mu \mathrm{m}$ with no other objects in the field and was registered with respect to the western cluster in Figure $1 a$ by assuming its peak emission coincides with the $2.2 \mu \mathrm{m}$ and $8.4 \mathrm{GHz}$ peaks.

The emission seen at $12.5 \mu \mathrm{m}$ is localized into five distinct emission centers at the locations of $\mathrm{A}, \mathrm{B} 1, \mathrm{~B} 2, \mathrm{C}$, and $\mathrm{C}^{\prime}$. The total flux density from the sum of the individual components is reported in Table 2 and shows that the total flux density measured at $12 \mu \mathrm{m}$ by $I R A S$ is accounted for by the total of these components as measured in the ground-based observations. Thus, at least at $12 \mu \mathrm{m}$, there is negligible 


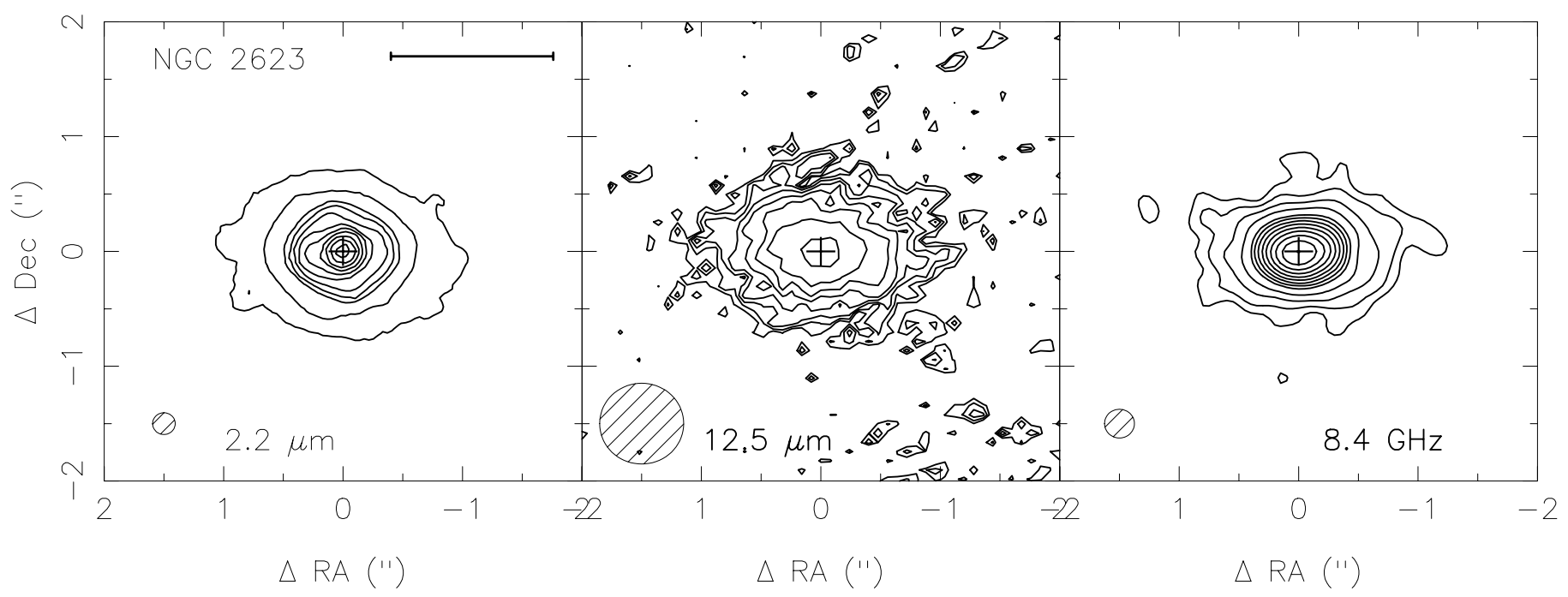

FIG. $3 a$
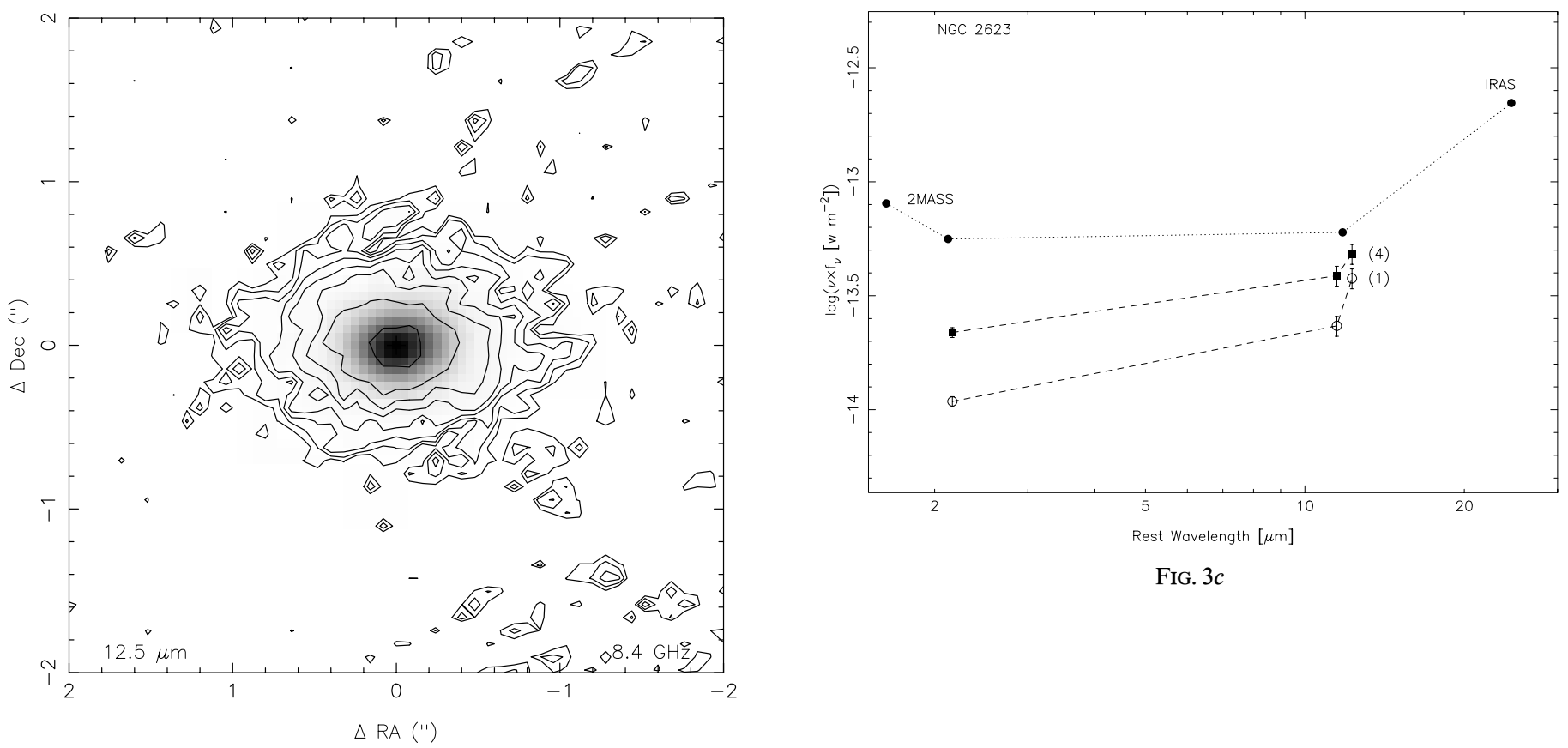

FIG. $3 c$

FIG. $3 b$

FIG. 3. (a) Montage of contour plots of NGC 2623 at $2.2 \mu \mathrm{m}, 12.5 \mu \mathrm{m}$, and $8.4 \mathrm{GHz}$. The origin is chosen to be at the peak emission in the $2.2 \mu \mathrm{m}$ image. The astrometric registration of the images at the different wavelengths is discussed in the text. The location of the center in each image is indicated by a plus sign in each panel. (b) Contour map of NGC 2623 at $12.5 \mu \mathrm{m}$ overlaid on the corresponding gray-scale image of $8.4 \mathrm{GHz}$ emission. (c) Spectral energy distribution of NGC 2623 . The filled circles represent the integrated fluxes taken from IRAS and 2MASS data. The aperture photometry in a 4 " diameter beam is represented by filled squares, the photometry in a 1" diameter beam is represented by open circles. The $2.2 \mu$ m photometric data are scaled from (S00).

luminosity, in comparison with these sources, being emitted in the many other supergiant $\mathrm{H}$ II regions in this system. A similar conclusion has been reached by Alonso-Herrero et al. (2000) based on the near-infrared NICMOS observations of this galaxy.

A direct comparison between the $12.5 \mu \mathrm{m}$ and $8.4 \mathrm{GHz}$ maps of NGC 3690 is shown in Figure 4c, where the contours of the $12.5 \mu \mathrm{m}$ image are overlaid on the gray scale of the $8.4 \mathrm{GHz}$ image of C91. Sources B1, C, and $\mathrm{C}^{\prime}$ agree very well, while there is no apparent radio counterpart for the faint $12.5 \mu \mathrm{m}$ source B2. The $12.5 \mu \mathrm{m}$ sources are quite compact; the Keck data have a resolution of 0".6 (125 pc), and only source $\mathrm{C}$ is clearly resolved in the Keck imaging.
While the vast majority of the infrared luminosity is produced by the five sources isolated at $12.5 \mu \mathrm{m}$, there is a low level of thermal infrared emission that is detected clearly in the $3.2 \mu \mathrm{m}$ image. In Figure $4 d$, the $3.2 \mu \mathrm{m}$ image is stretched to show the low surface brightness emission and, for comparison, the $1.644 \mu \mathrm{m}$ [Fe II] image from AlonsoHerrero et al. (2000). There is very low level $3.2 \mu \mathrm{m}$ emission seen tracing the $\mathrm{H}$ in regions from source $\mathrm{B} 2$ to $\mathrm{C}$, as well as the $\mathrm{H}$ II regions surrounding the nucleus in the eastern galaxy (source $\mathrm{A}=\mathrm{IC} 694$ ).

All of the sources detected at $12.5 \mu \mathrm{m}$ are seen in the NICMOS $2.2 \mu \mathrm{m}$ continuum images. Source $\mathrm{C}^{\prime}$ is inconspicuous in the near-infrared image. This source has the 


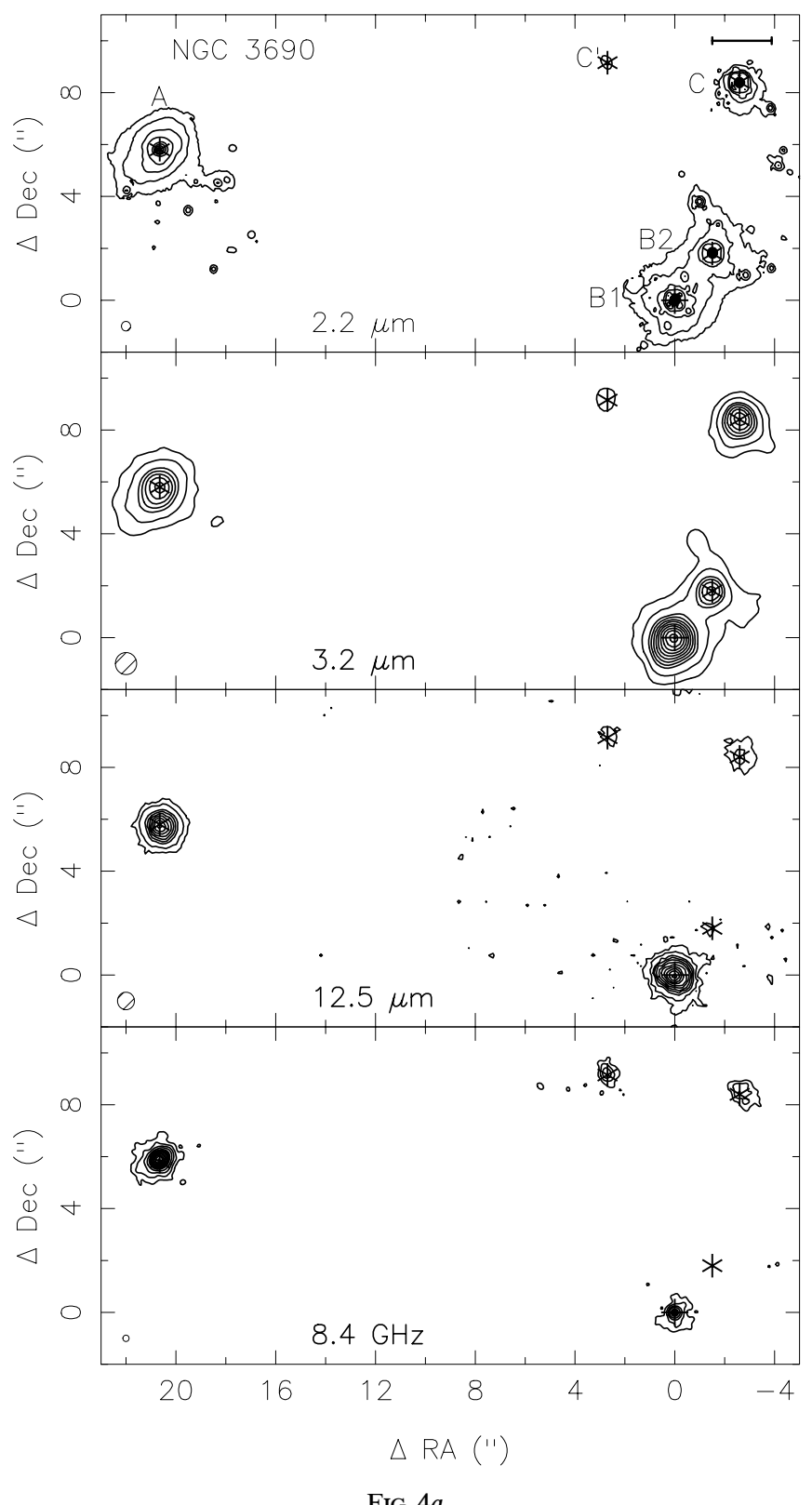

FIG. 4.-(a) Montage of contour plots of NGC 3690/IC 694 at $2.2 \mu \mathrm{m}, 3.2 \mu \mathrm{m}, 12.5 \mu \mathrm{m}$, and $8.4 \mathrm{GHz}$. The positions of sources A, B1, B2, C, and C', as determined at $2.2 \mu \mathrm{m}$, are indicated in each panel. The astrometric registration of the images at the different wavelengths is discussed in the text. The $2.2 \mu \mathrm{m}$ image is from Alonso-Herrero et al. (2000) and the $3.2 \mu \mathrm{m}$ image is from NIRC. (b) Montage of contour plots of NGC 3690 at $2.2 \mu \mathrm{m}, 3.2 \mu \mathrm{m}, 12.5 \mu \mathrm{m}$, and 8.4 GHz. This is an expanded scale of the western portion of $(a)$, showing in more detail the sources in NGC 3690, but otherwise the same. (c) Contour map of NGC 3690 at $12.5 \mu \mathrm{m}$ overlaid on the corresponding gray-scale image of $8.4 \mathrm{GHz}$ emission. (d) Gray-scale images of NGC 3690/IC 694 at $3.2 \mu \mathrm{m}$ and in the [Fe II $1.644 \mu \mathrm{m}$ emission line plus continuum (from Alonso-Herrero et al. 2000). Both images has been stretched to enhance the low-level emission. (e) Spectral energy distributions of sources in NGC 3690/IC 694. The integrated fluxes ( filled circles) are taken from IRAS and 2MASS data. The filled squares represent photometry in a 2".5 diameter beam centered on sources A, B1, C, and $\mathrm{C}^{\prime}$ and a 2" diameter beam centered on source B2, as shown in $(a)$ and $(b)$. The filled triangles represent the sum of the photometric points for the individual sources using a $4^{\prime \prime}$ beam for sources A, B1, C, and C' and a $2^{\prime \prime}$ beam for B2. The $3.2 \mu \mathrm{m}$ data are from NIRC, while the $2.2 \mu \mathrm{m}$ data for the small beams are from NICMOS (Alonso-Herrero et al. 2000). (f) Spectral energy distributions of individual sources in NGC 3690/IC 694. The filled triangles represent the sum of all the photometric points for the individual sources and are presented for reference in each panel. In the top panel are data for source A in 1" (filled squares) and 2".5 (open squares) diameter beams. The middle panel presents data for sources B1 (1" diameter beam, open squares; 2.5 diameter beam, filled squares) and B2 (1" diameter beam, open squares; $2^{\prime \prime}$ diameter beam, filled stars). The bottom panel presents data for sources $\mathrm{C}$ (1" diameter beam, open squares, 2".5 diameter beam, filled squares) and $\mathrm{C}^{\prime}$ (1" diameter beam, open squares; $2^{\prime \prime} .5$ diameter beam, filled squares). The data sources are as in $(e)$.

largest ratio of $S_{v}(12 \mu \mathrm{m}) / S_{v}(2.2 \mu \mathrm{m})$ of those detected at $12.5 \mu \mathrm{m}$, being more than 5 times brighter at $12.5 \mu \mathrm{m}$ normalized to the $2.2 \mu \mathrm{m}$ flux density than either sources A or $\mathrm{B} 1$, the brightest $12.5 \mu \mathrm{m}$ sources. Alonso-Herrero et al. (2000) do not identify this as the source having the greatest extinction. This suggests that the more obscured sources (e.g., A, B1, C) have luminous stellar contributions associ- ated with but separate from the highly infrared-luminous regions.

Figures $4 e$ and $4 f$ show the spectral energy distributions of the various components. Figure $4 e$ displays the SEDs of the integrated light from the entire system, the sum of $4^{\prime \prime}$ diameter beams centered on A, B1, C, and $\mathrm{C}^{\prime}$, and a $2^{\prime \prime}$ diameter beam centered on B2, and the individual SEDs in 


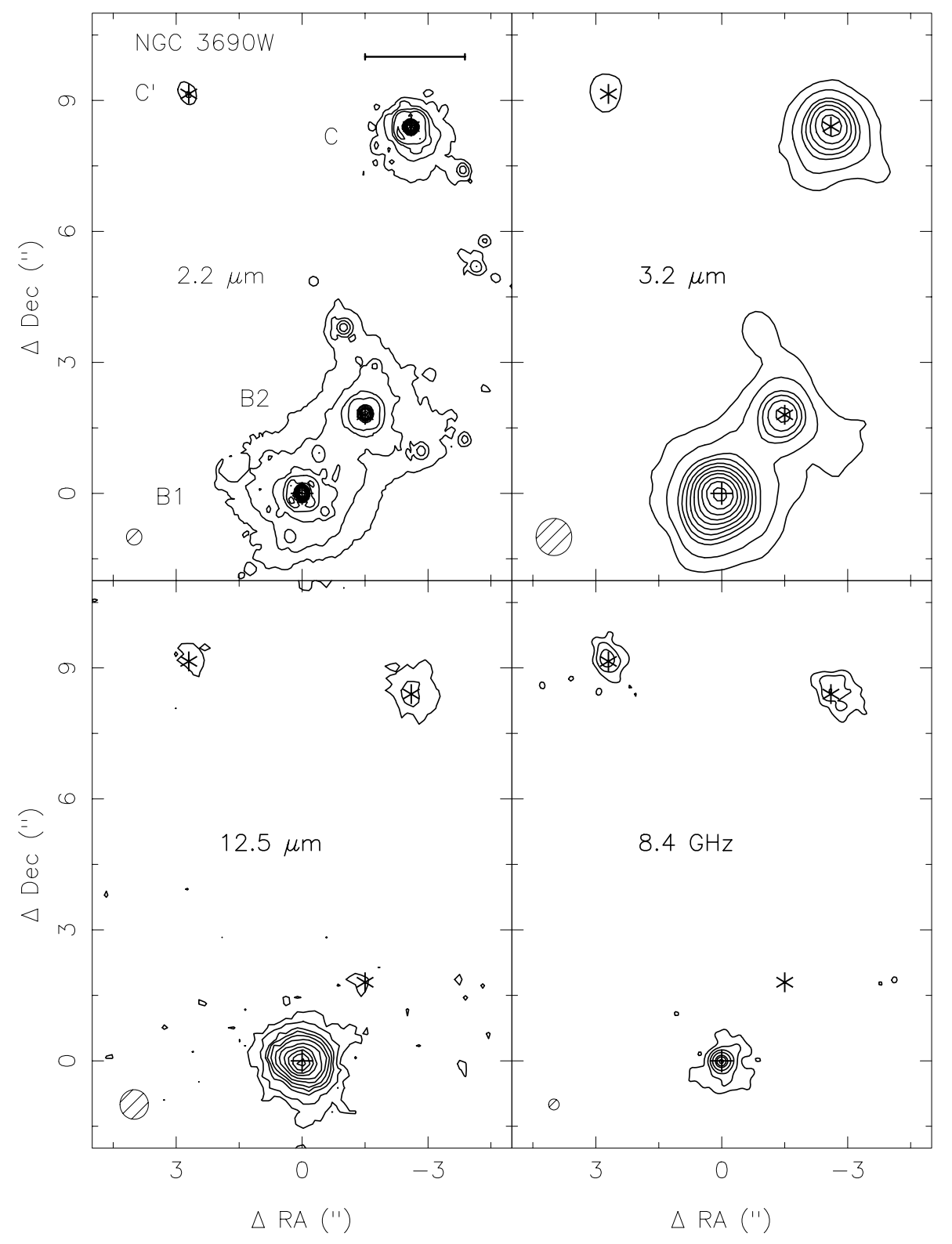

FIG. $4 b$

2 2.5 beams of the five separate peaks. Figure $4 f$ presents three panels that show the SEDs of sources A, B, and C separately in $1^{\prime \prime}$ and 2.5 diameter beams. The $2.2 \mu \mathrm{m}$ data are derived from NICMOS observations (Alonso-Herrero et al. 2000), while the $3.2 \mu \mathrm{m}$ data are from NIRC observations shown in Figure 4d.

As mentioned above, the total flux density measured in the $12.5 \mu \mathrm{m}$ images accounts for virtually all the flux density measured by IRAS (Soifer et al. 1989). The spectroscopy of Dudley (1999) shows that each of the separate sources, A, $\mathrm{B} 1, \mathrm{C}$, and $\mathrm{C}^{\prime}$, includes a contribution from PAH emission, while sources $\mathrm{B} 1$ and $\mathrm{A}$ also show overlying silicate absorption. The difficulty in determining the contribution of PAH emission to the photometry is illustrated by the comparison sources $\mathrm{A}$ and $\mathrm{C}$ in Figure $4 d$. Source $\mathrm{A}$ has an apparent small PAH contribution, but the spectrum of Dudley reveals that there is a substantial PAH emission feature superposed on a steeply rising spectrum, while the similar strength feature seen in the SED of source C, which is sug- gestive of PAH emission in the photometry, is a result of being superposed on a flatter SED for this source.

\section{5. $I C 883(=U G C 8387=\operatorname{Arp~193)}$}

IC 883 (=UGC $8387=$ Arp 193) is a disk galaxy with crossed tails in optical images suggestive of a merger. At a redshift of $c z=7000 \mathrm{~km} \mathrm{~s}^{-1}\left(92 \mathrm{Mpc}, 470 \mathrm{pc} \mathrm{arcsec}^{-1}\right)$, the infrared luminosity of this system is $3 \times 10^{11} L_{\odot}$ (Soifer et al. 1987). Its optical spectrum (Villeux et al. 1995) is classified as that of a LINER, while its mid-infrared spectrum shows PAH emission (Dudley 1999). High-resolution NICMOS observations of the bright central portion of this system reveal an apparently edge-on disk galaxy with a significant dust lane (S00). High-resolution radio observations show a source extended along the infrared disk with a size of $\sim 4^{\prime \prime} \times 0$ ".7 (C91).

Figure $5 a$ shows the $12.5 \mu \mathrm{m}$ Keck image, along with the $2.2 \mu \mathrm{m}$ NICMOS image from $\mathrm{S} 00$ and the $8.4 \mathrm{GHz}$ radio image from C91. The $12.5 \mu \mathrm{m}$ image of Figure $5 a$ is 


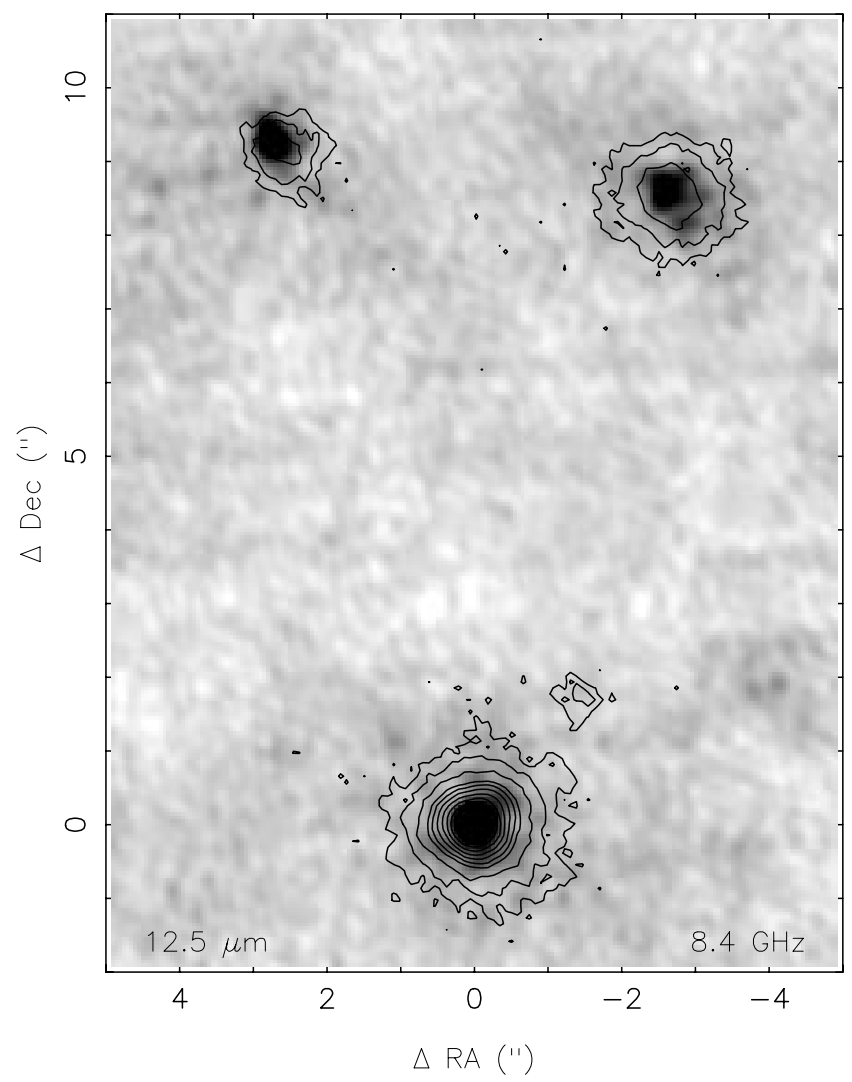

FIG. $4 c$

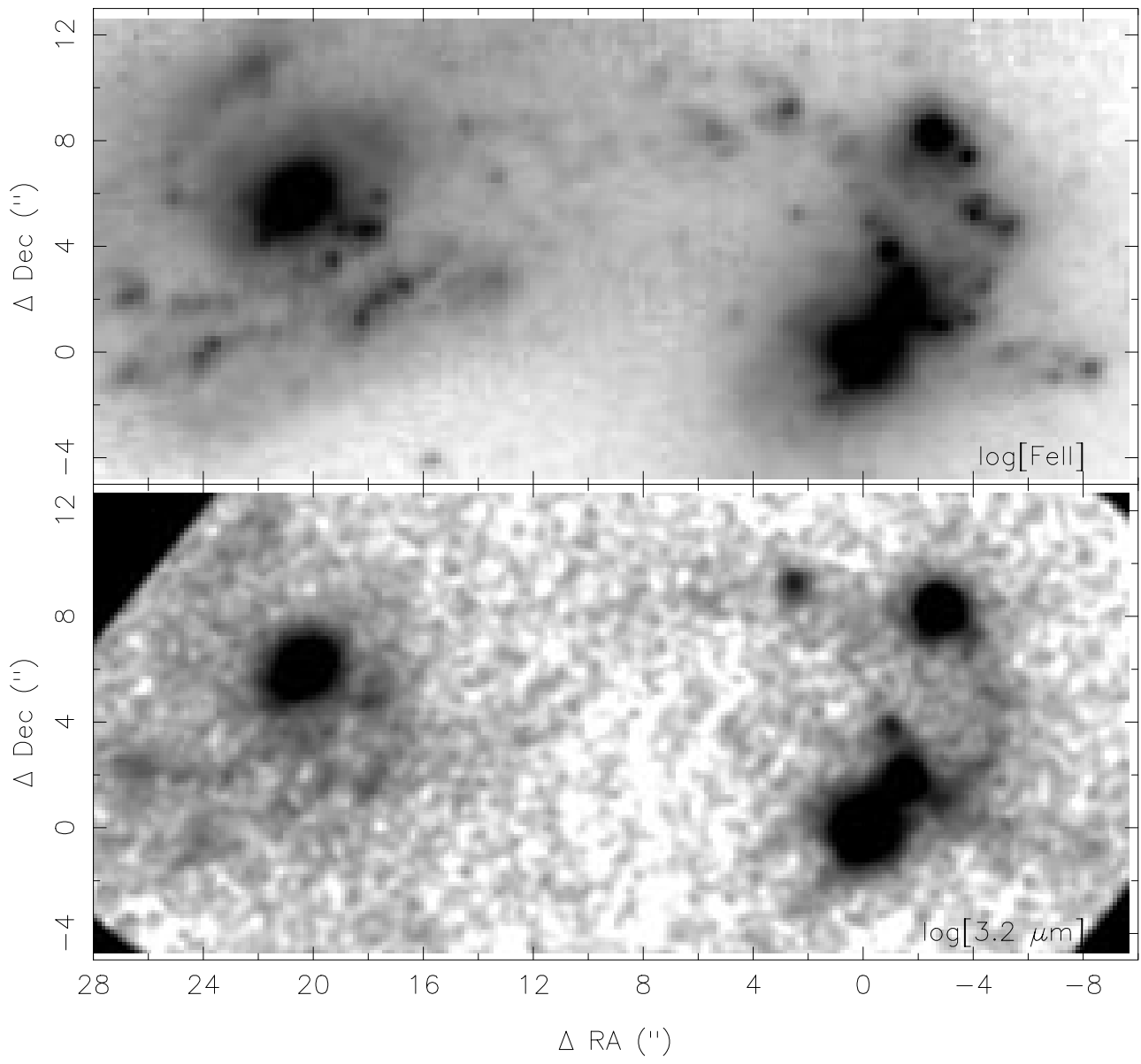

FIG. $4 d$ 


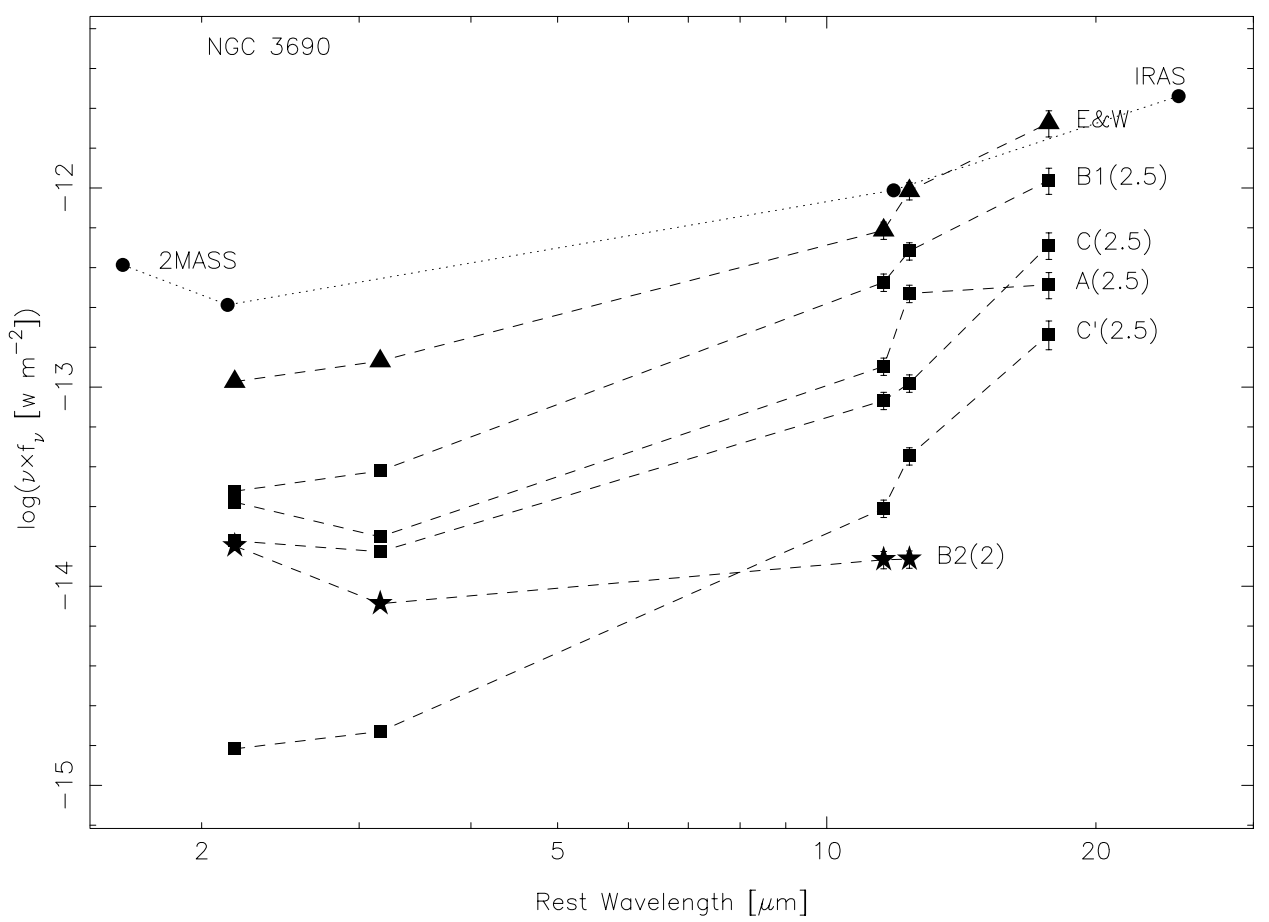

FIG. $4 e$

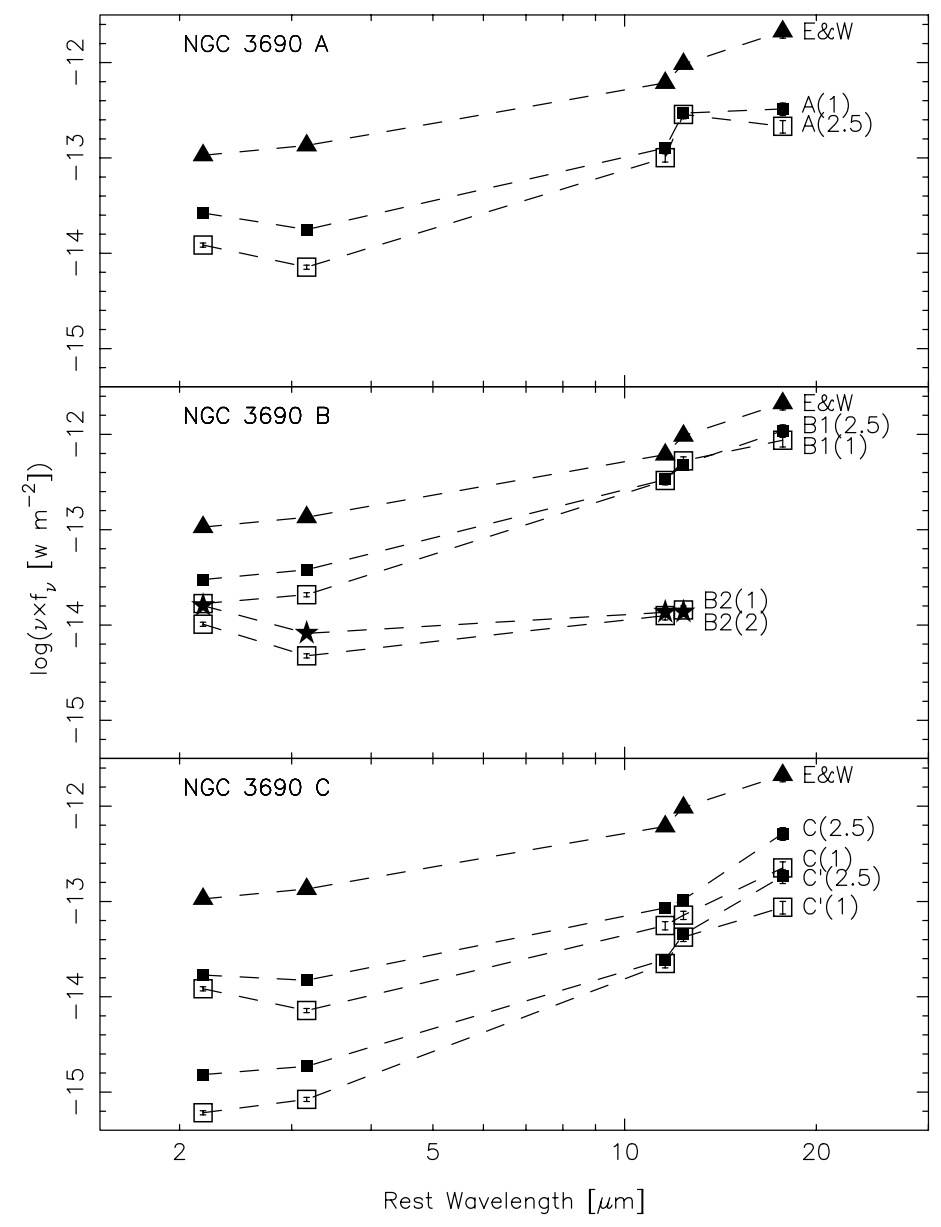

FIG. $4 f$ 


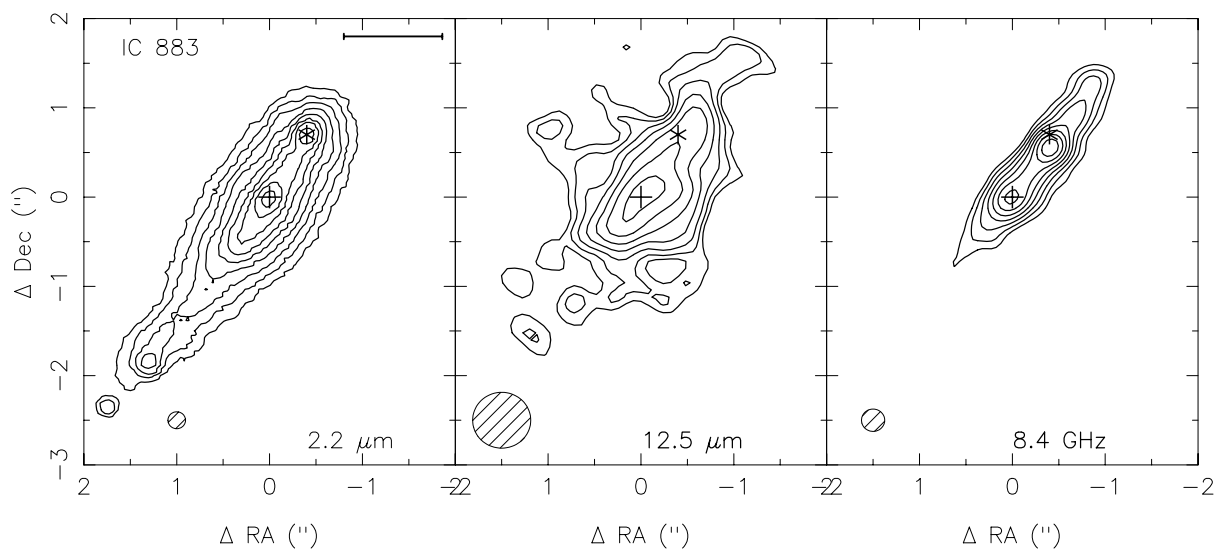

FIG. $5 a$

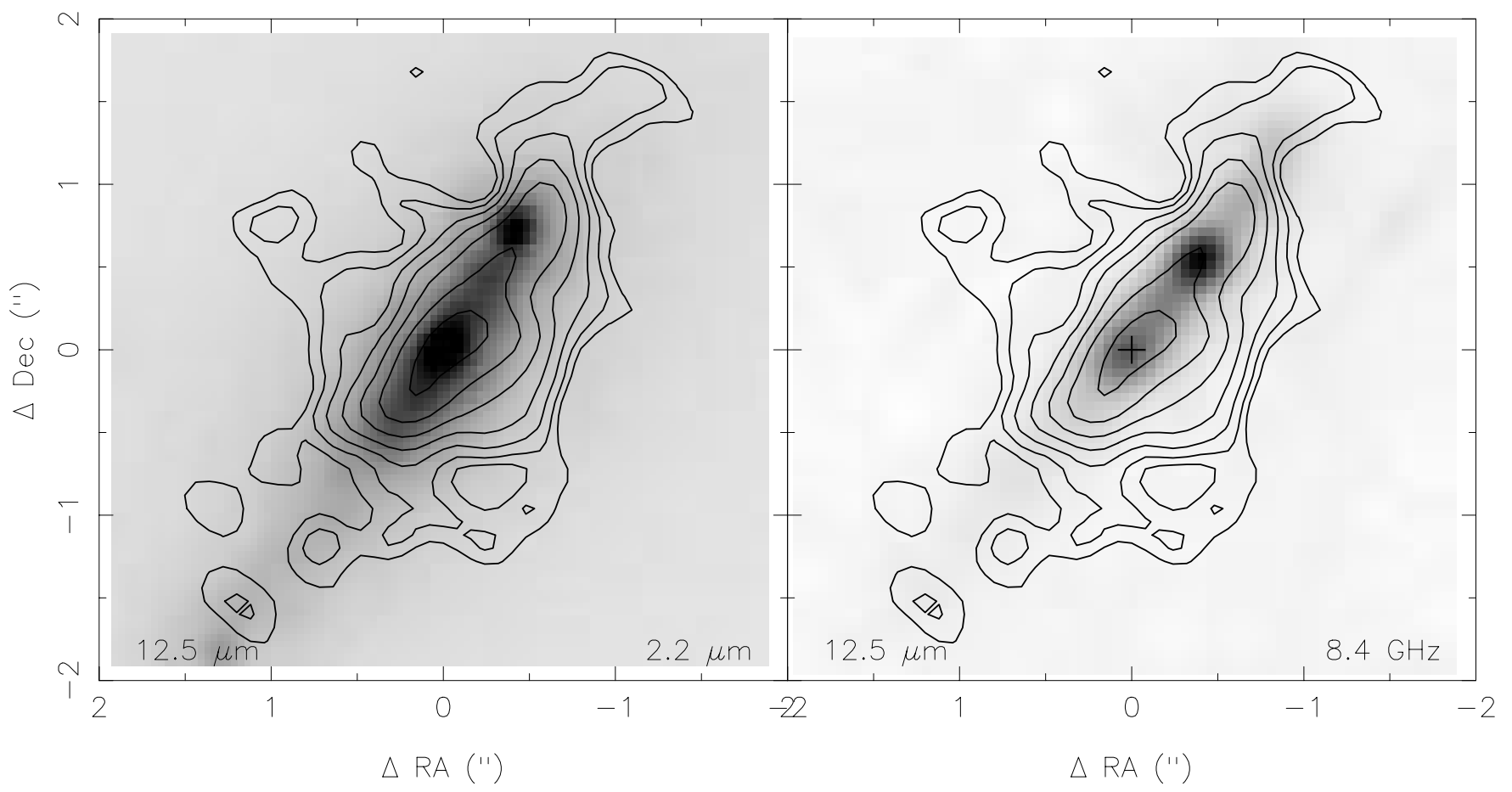

FIG. $5 b$

Fig. 5. - (a) Montage of contour plots of IC 883 at $2.2 \mu \mathrm{m}, 12.5 \mu \mathrm{m}$, and $8.4 \mathrm{GHz}$. The origin is chosen to be at the peak emission in the $2.2 \mu \mathrm{m}$ image. The astrometric registration of the images at the different wavelengths is discussed in the text. The location of the two brightest peaks at $2.2 \mu \mathrm{m}$ are indicated in each image by a plus sign and an asterisk at the same relative location in each panel. (b) Overlay of the $12.5 \mu$ m emission contours of IC 883 on the corresponding gray-scale image at $2.2 \mu \mathrm{m}$ is presented in the left panel. A contour map at $12.5 \mu \mathrm{m}$ is overlaid on the corresponding gray-scale image of 8.4 $\mathrm{GHz}$ emission in the right panel. (c) Spectral energy distribution of IC 883 is shown. The filled circles represent the integrated fluxes taken from IRAS and 2MASS data. The aperture photometry in a 4 " diameter beam is represented by filled squares; the photometry in a 1 " diameter beam is represented by open circles. The $2.2 \mu \mathrm{m}$ photometric data are scaled from the NICMOS image (S00).

smoothed to 0.62 resolution from the original 0 ".40 to improve the $\mathrm{S} / \mathrm{N}$, while the resolution is 0.19 at $2.2 \mu \mathrm{m}$ and 0 ".24 at $8.4 \mathrm{GHz}$. Two peaks visible at $2.2 \mu \mathrm{m}$ are marked; the marks are reproduced at the same relative positions in all three panels of Figure $5 a$.

The astrometric registration of the radio and $12.5 \mu \mathrm{m}$ images is based on the assumption that the $12.5 \mu \mathrm{m}$ peak coincides with the southeastern radio peak. This forces general agreement between the radio and $12.5 \mu \mathrm{m}$ structures. The $2.2 \mu \mathrm{m}$ image was registered with respect to the radio image by assuming coincidence of the central peak at $2.2 \mu \mathrm{m}$ and the southeastern radio peak.

The $12.5 \mu \mathrm{m}$ image shows that the emission is extended along a position angle of $135^{\circ}$ and shows a significant exten- sion perpendicular to the major axis. The measured $12.5 \mu \mathrm{m}$ FWHM is $1.8 \times 0$ ". 7 , while the full extent of the $12.5 \mu \mathrm{m}$ emission is $3^{\prime \prime} \times 1^{\prime \prime} .7$. The $8.4 \mathrm{GHz}$ image shows a similar size along the major axis with significantly less extent perpendicular to this axis, having a full extent along the minor axis of 0.7 . With the assumption that the southeastern radio peak coincides with the peak of the $12.5 \mu \mathrm{m}$ emission, the northwest radio peak appears to coincide with a shoulder of $12.5 \mu \mathrm{m}$ emission. This is illustrated in Figure $5 b$ where the $12.5 \mu \mathrm{m}$ contours are overlaid on a gray scale of the 8.4 $\mathrm{GHz}$ image. The emission perpendicular to the major axis appears significantly more extended at $12.5 \mu \mathrm{m}$ than at 8.4 $\mathrm{GHz}$, but this could be a result of the lack of sensitivity to low surface brightness emission in the radio image. 


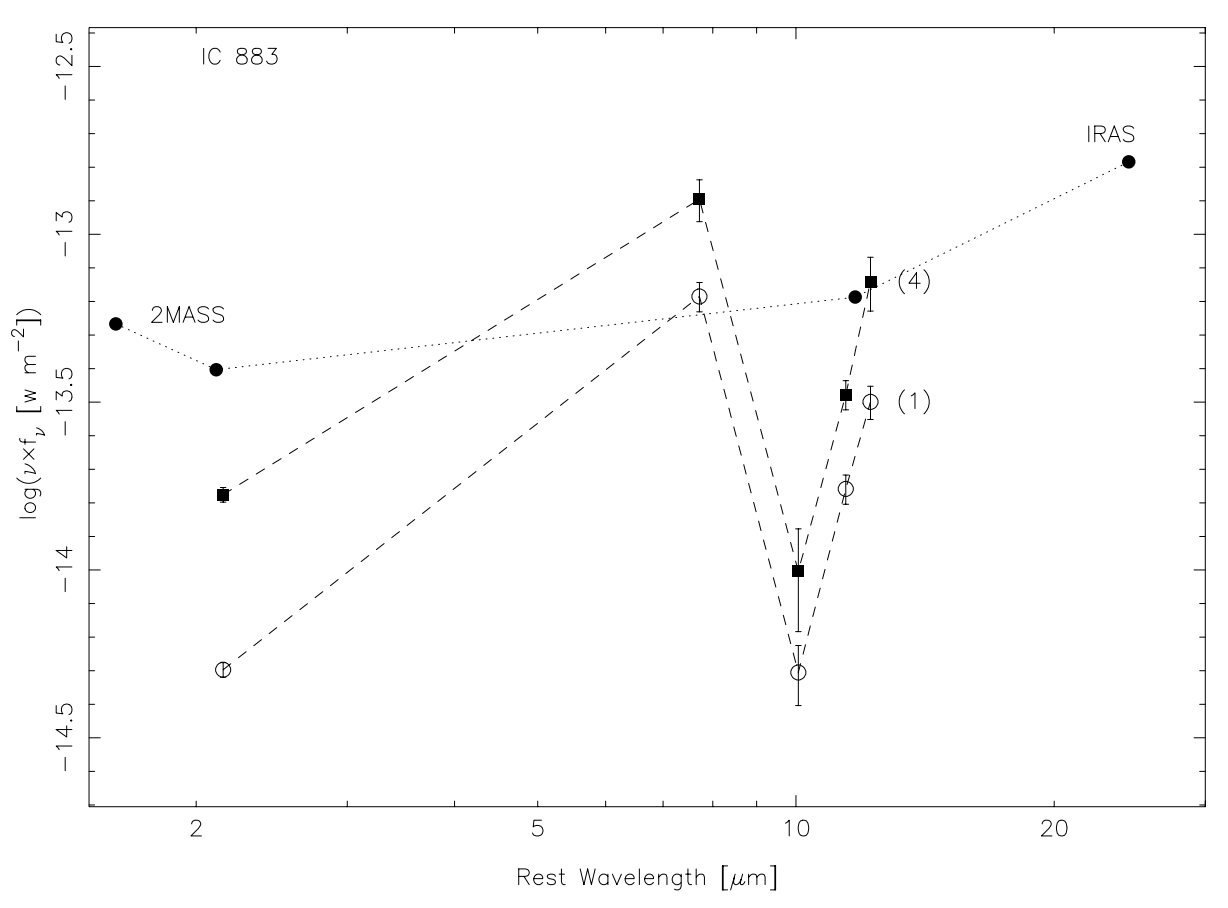

FIG. $5 c$

The $2.2 \mu \mathrm{m}$ image does not agree in morphology particularly well with the mid-infrared and radio images. Again we have assumed that the central peak of $2.2 \mu \mathrm{m}$ emission coincides with the radio and infrared peaks and present in Figure $5 b$ a gray-scale image of the NICMOS $2.2 \mu \mathrm{m}$ image with $12.5 \mu \mathrm{m}$ contours overlaid. The $2.2 \mu \mathrm{m}$ peak to the northwest of the center differs in position relative to the radio peak by 0.2 or $90 \mathrm{pc}$ at the distance of the galaxy. In addition, the $2.2 \mu \mathrm{m}$ extent is significant to the southeast of the central peak, while both the mid-infrared and $8.4 \mathrm{GHz}$ images show extent to the northwest of the central peak.

In addition to imaging IC 883 at $12.5 \mu \mathrm{m}$, Keck images were obtained at 8.0, 10.3, and $11.7 \mu \mathrm{m}$. These images showed virtually identical morphology to that shown in Figure 5a. Based on one-dimensional profiles of flux versus position from each Keck image, there is no discernible variation in the SED of IC 883 along its major axis at a resolution of 0 ".6 $(270 \mathrm{pc})$ over the central $3^{\prime \prime}$ or $1.4 \mathrm{kpc}$. The spectral energy distribution of IC 883 is shown in Figure $5 c$. The comparison of the Keck observations and the IRAS data shows that the Keck observations account for all of the mid-infrared emission detected by IRAS in this system. The comparison of the $1^{\prime \prime}$ and $4^{\prime \prime}$ photometry illustrates the point that the SED does not vary significantly with beam diameter.

The Keck data show a strong drop in flux at $10 \mu \mathrm{m}$, indicative of significant silicate absorption affecting the emergent spectrum. The mid-infrared spectrum of Dudley (1999) shows strong PAH emission with a depression that is not nearly as strong as presented here. This apparent discrepancy is likely due to the low $\mathrm{S} / \mathrm{N}$ in the Dudley observations at $\sim 10 \mu \mathrm{m}$.

\section{6. $N G C 6090(=U G C 10267)$}

NGC 6090 (=UCG 10267), at a redshift $c z=8785 \mathrm{~km}$ $\mathrm{s}^{-1}\left(117 \mathrm{Mpc}, 590 \mathrm{pc} \operatorname{arcsec}^{-1}\right)$, has an infrared luminosity of $3 \times 10^{11} L_{\odot}$ (Soifer et al. 1987). The visual image shows a face-on spiral with a close companion separated by $5^{\prime \prime}$ or $\sim 3 \mathrm{kpc}$ to the southwest and faint tidal tails extended over $\sim 2^{\prime}(72 \mathrm{kpc})$. The optical spectrum is classified as $\mathrm{H}$ II-like (Veilleux et al. 1995). High-resolution VLA imaging at 8.4 $\mathrm{GHz}$ (C91) shows a compact source, while a lower resolution map at $1.4 \mathrm{GHz}$ (Condon et al. 1990) shows emission extending over the face of both the spiral galaxy and the close companion. NICMOS observations have been reported for the NGC 6090 system by S00 and Dinshaw et al. (1999). Bryant \& Scoville (1999) found that the molecular gas in this system is located approximately midway between the two galaxies.

The $12.5 \mu \mathrm{m}$ image of NGC 6090 is presented in Figure $6 a$, along with the $2.2 \mu \mathrm{m}$ image from $\mathrm{S} 00$, an $8.4 \mathrm{GHz}$ image reprocessed from the data of C91 with angular resolution of 0 .'5, and the $\mathrm{Br} \gamma$ image from Soifer et al. (2001). The astrometric registration of the radio and $12.5 \mu \mathrm{m}$ images is based on the assumption that the brightest 12.5 $\mu \mathrm{m}$ peaks coincide with radio peaks with the same angular separation (and position angle). The mid-infrared image was located with respect to the near-infrared continuum through the $\mathrm{Br} \gamma$ image. Because of the strong similarity in the morphological structure between the $12.5 \mu \mathrm{m}$ and $\mathrm{Br} \gamma$ images, the $12.5 \mu \mathrm{m}$ emission peaks were assumed to coincide with corresponding peaks in the $\mathrm{Br} \gamma$ image. The $\mathrm{Br} \gamma$ image was obtained using the Palomar Integral Field Spectrograph (PIFS; Murphy et al. 1999) and was obtained simultaneously with a $2.2 \mu \mathrm{m}$ continuum image of this galaxy. The $2.2 \mu \mathrm{m}$ continuum image obtained with PIFS corresponds well with the $2.2 \mu \mathrm{m}$ NICMOS image, so that the $\mathrm{Br} \gamma$ and $2.2 \mu \mathrm{m}$ continuum images are located very precisely $(<0$ ".08) with respect to each other. The PIFS data will be discussed in more detail in Soifer et al.

In addition to the morphological similarities between the $12.5 \mu \mathrm{m}$ and $\mathrm{Br} \gamma$ images, there is a strong physical reason for expecting the emission to be spatially coincident; the currently ionized gas traces the ionizing stars and the mid- 


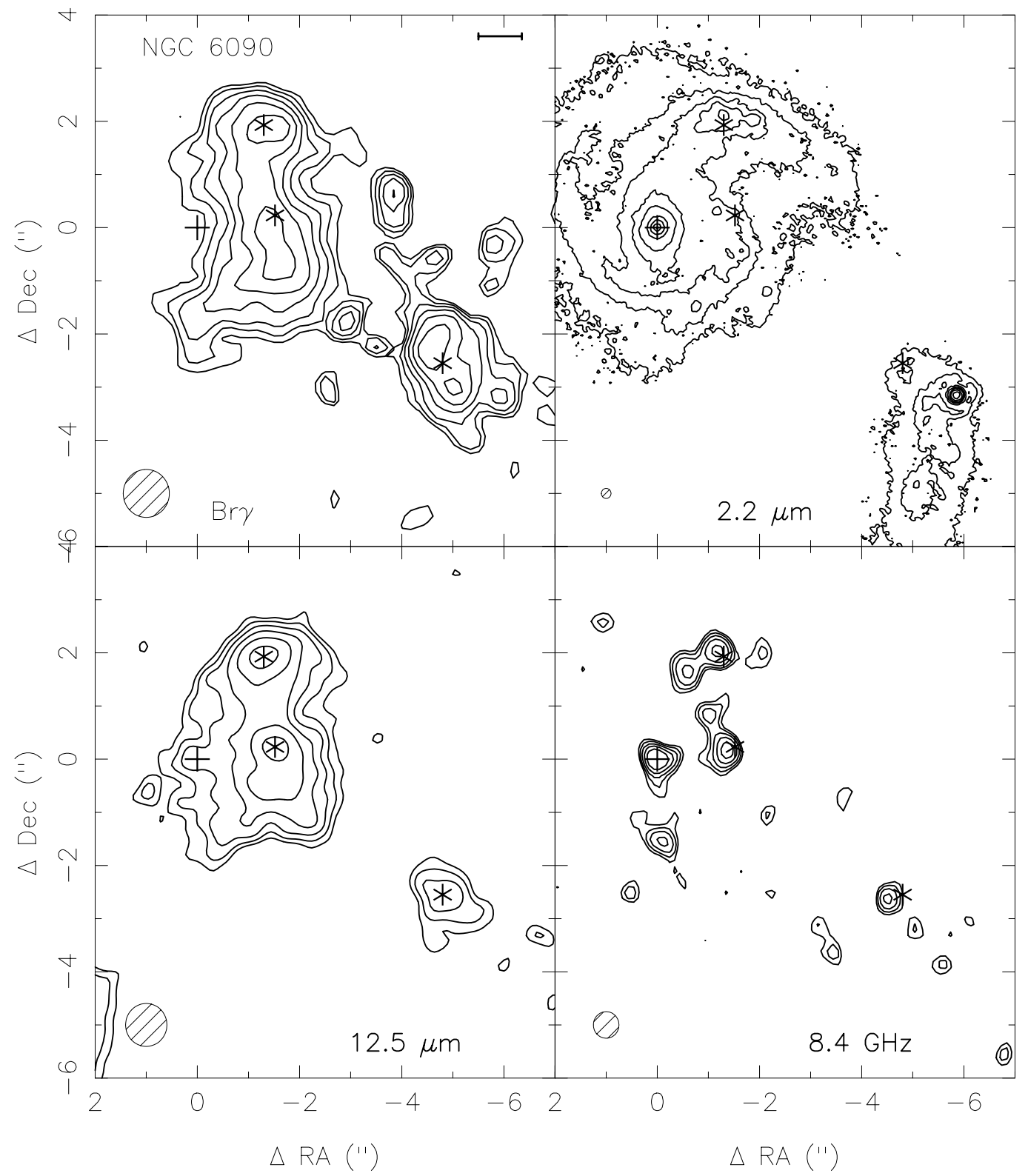

FIG. $6 a$

Fig. 6.-(a) Montage of contour plots of NGC 6090 at $\mathrm{Br} \gamma(2.16 \mu \mathrm{m}), 2.2 \mu \mathrm{m}, 12.5 \mu \mathrm{m}$, and $8.4 \mathrm{GHz}$. The origin is chosen to be at the nucleus of the face-on spiral galaxy in the $2.2 \mu \mathrm{m}$ image and is indicated by a plus sign in each panel. The astrometric registration of the images at the different wavelengths is discussed in the text. The location of a secondary peak at $2.2 \mu \mathrm{m}$ is indicated by an asterisk in each panel. The location of the secondary peak in the $8.4 \mathrm{GHz}$ emission is indicated by a times cross in each panel. The Bry image was obtained using the Palomar Integral Field Spectrograph (PIFS; Murphy et al. 1999). (b) Contour map of NGC 6090 at $12.5 \mu \mathrm{m}$ overlaid on the corresponding gray-scale image at $2.2 \mu \mathrm{m}$ (left) and the $8.4 \mathrm{GHz}$ image (right). (c) Spectral energy distributions of the sources in NGC 6090. The filled circles represent the integrated fluxes taken from IRAS and 2MASS data. The filled squares represent photometry in a $6^{\prime \prime}$ diameter beam centered on the eastern source. The open squares and open triangles represent photometry in a $1 . .5$ diameter beam centered on the southern and northern components of the eastern source. The open circles and filled squares marked with a " $\mathrm{W}$ " present photometry in 1".5 and 4 " diameter beams centered on the western source. The $2.2 \mu$ m photometric data are scaled from the NICMOS image (S00).

infrared emission traces the dust heated by these (presumably) same stars.

With the assumption that the $12.5 \mu \mathrm{m}$ and $\mathrm{Br} \gamma$ images coincide, a faint $12.5 \mu \mathrm{m}$ peak to the east of the brightest regions in the image coincides with the $2.2 \mu \mathrm{m}$ nucleus in the face-on spiral NGC 6090. This is shown directly in Figure $6 b$, where the $12.5 \mu \mathrm{m}$ contours are overlaid on a gray scale of the $2.2 \mu \mathrm{m}$ image. The main emission at 12.5 $\mu \mathrm{m}$ coincides with regions of blue, unresolved sources in the spiral arms in the face-on spiral galaxy as noted by S00. In addition, the faint $12.5 \mu \mathrm{m}$ emission $\sim 3^{\prime \prime}$ south and east of the bright $12.5 \mu \mathrm{m}$ emission is located on a minor peak (not the bright point source) in the nearby companion galaxy. This region is spatially coincident with additional blue unresolved sources in the companion galaxy (S00). The bright unresolved source in the companion galaxy does not coincide with any radio or mid-infrared emission, consistent with the suggestion that it is a foreground object. The brightest mid-infrared peaks are extended by $\sim 1^{\prime \prime}$, or 600 pc, while the whole $12.5 \mu \mathrm{m}$ emitting region in NGC 6090 is extended over $4^{\prime \prime} \times 2^{\prime \prime}$, or $2.4 \times 1.2 \mathrm{kpc}$.

With the astrometric registrations as presented in Figure $6 a$, the agreement between the peaks of $8.4 \mathrm{GHz}$ emission and $12.5 \mu \mathrm{m}$ emission is quite good. Each peak of $12.5 \mu \mathrm{m}$ 


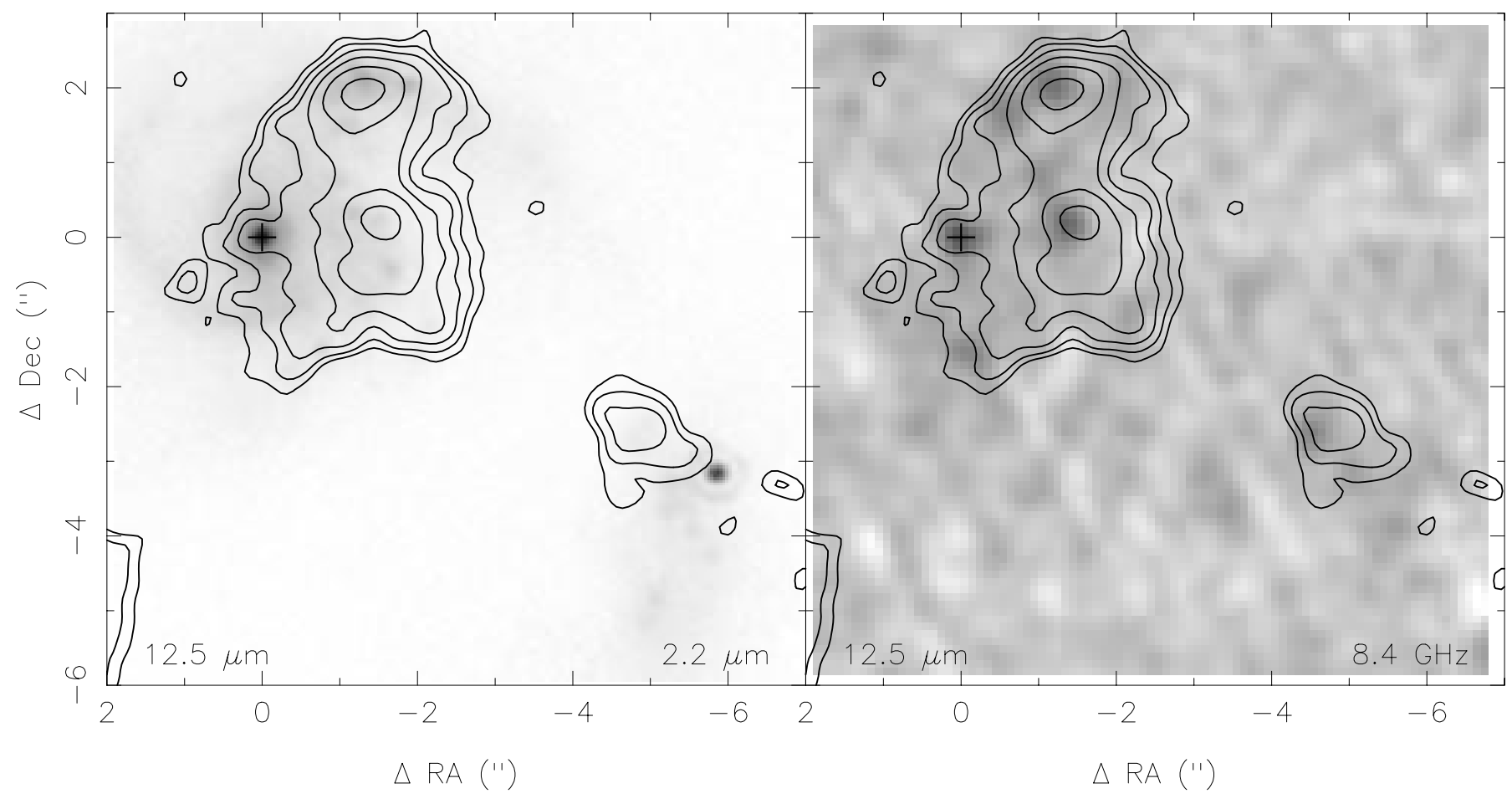

FIG. $6 b$

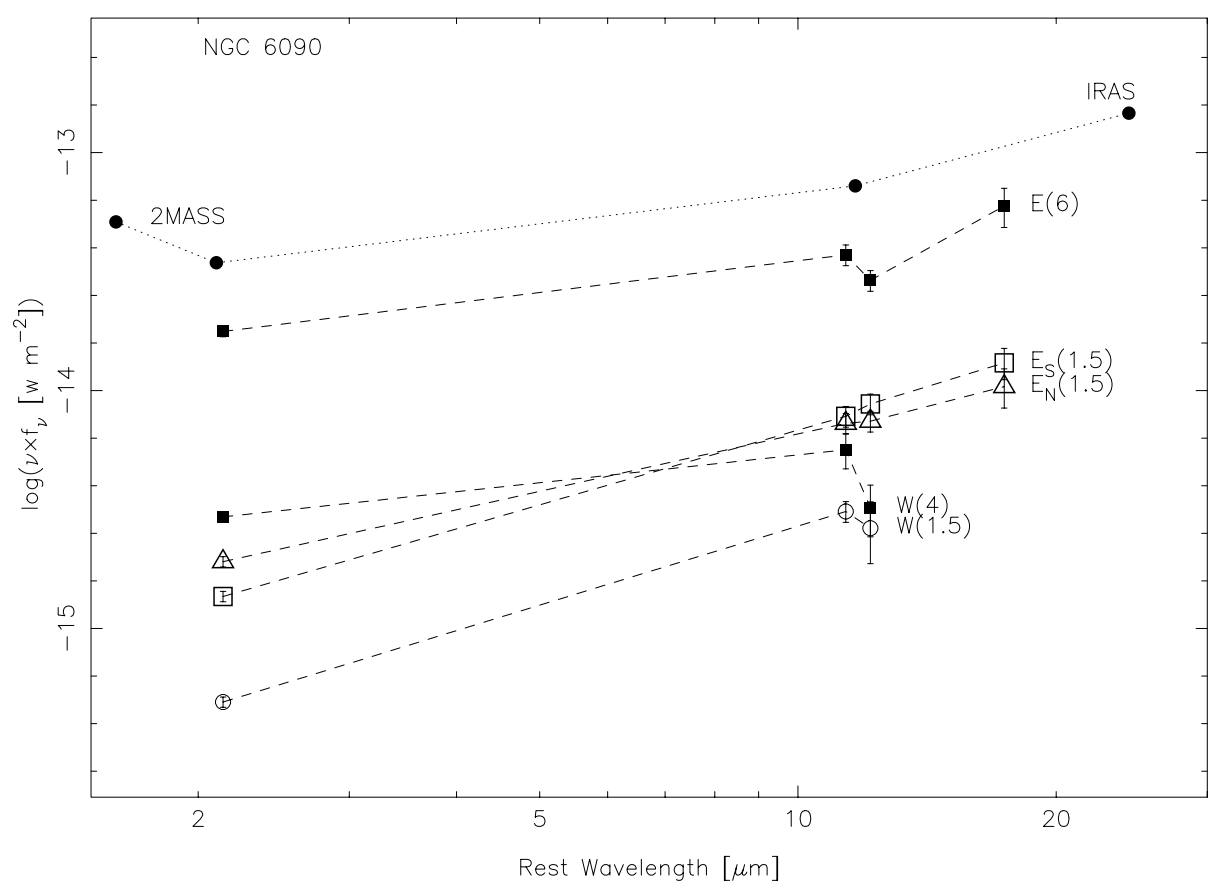

Fig. $6 c$

emission corresponds to a peak of radio emission (with perhaps a $0^{\prime \prime} .2$ discrepancy between the faint $12.5 \mu \mathrm{m}$ peak to the southeast and the corresponding radio peak). In addition, there appears to be a radio peak at the position of the near-infrared nucleus of NGC 6090.

In NGC 6090, the galaxy nucleus is at best a minor source of infrared luminosity. The current location of luminous star formation appears to be in the spiral arms of the face-on galaxy and the portions of the companion galaxy closest to the spiral. These regions are within spiral arms and close to the molecular gas which lies between the two galaxies (Bryant \& Scoville 1999). This suggests that the molecular gas remained behind after the passage of the two galaxies on a closely interacting trajectory, and the current star formation was triggered in the locations of closest approach in the two galaxies.

Figure $6 c$ shows the spectral energy distributions of the peaks in NGC 6090, along with the large beam IRAS and 2MASS data. At $12 \mu \mathrm{m}$, approximately $50 \%$ of the flux density measured by $I R A S$ is detected in the LWS images. 
The substantial difference between the $12 \mu \mathrm{m}$ flux density measured by $I R A S$ and the integrated flux density measured at $12.5 \mu \mathrm{m}$ in the Keck image argues that there is significant diffuse mid-infrared emission in this system not detected in the ground-based imaging. Keck observations were obtained at 11.7, 12.5, and $17.9 \mu \mathrm{m}$. The increased flux density at $11.7 \mu \mathrm{m}$ compared with that at $12.5 \mu \mathrm{m}$, as seen in Figure $6 c$, suggests that there is strong PAH emission in this system. There is no published spectrum of NGC 6090 spanning this wavelength range.

\section{7. $\operatorname{Mrk} 331$ (=UGC 12812)}

Mrk 331 (=UCG 12812), at a redshift $c z=5500 \mathrm{~km} \mathrm{~s}^{-1}$ (73 Mpc, $370 \mathrm{pc} \operatorname{arcsec}^{-1}$ ), has an infrared luminosity of $2.5 \times 10^{11} L_{\odot}$ (Soifer et al. 1987). The visual image shows a high-surface brightness core with no obvious tidal tails. There are two galaxies of comparable brightness located within $2^{\prime}$. The optical spectrum is classified as $\mathrm{H}$ II-like (Veilleux et al. 1995), with a mid-infrared spectrum indicat- ing a combination of PAH emission and silicate absorption (Dudley 1999). High-resolution VLA imaging at $8.4 \mathrm{GHz}$ (C91) shows a bright central source surrounded by an elliptical ring of emission with dimensions $3^{\prime \prime} \times 2^{\prime \prime}$.

Figure $7 a$ shows the $12.5 \mu \mathrm{m}$ image of Mrk 331 along with images at $2.15 \mu \mathrm{m}\left(K_{\mathrm{s}}\right), 3.4 \mu \mathrm{m}$, and $8.4 \mathrm{GHz}$. A bright nucleus is seen at all wavelengths, as well as extended emission in an apparent disk or ring. In registering the images, it was assumed that the central peak was spatially coincident at all wavelengths.

At $8.4 \mathrm{GHz}$, the extended emission is distributed as a "ring" surrounding the bright nucleus. At $12.5 \mu \mathrm{m}$, there is a bright nucleus that is unresolved in the 0.3 seeing, as well as emission that appears to have a similar structure as the radio ring. The $12.5 \mu \mathrm{m}$ emission appears to be distributed over the full disk of the system, extending over $\sim 4^{\prime \prime}$ or 1.5 kpc diameter, with a "barlike" structure extending from the nucleus to the northeast. The image of Mrk 331 obtained at $11.7 \mu \mathrm{m}$ is virtually identical to that at $12.5 \mu \mathrm{m}$. At $8.4 \mathrm{GHz}$,

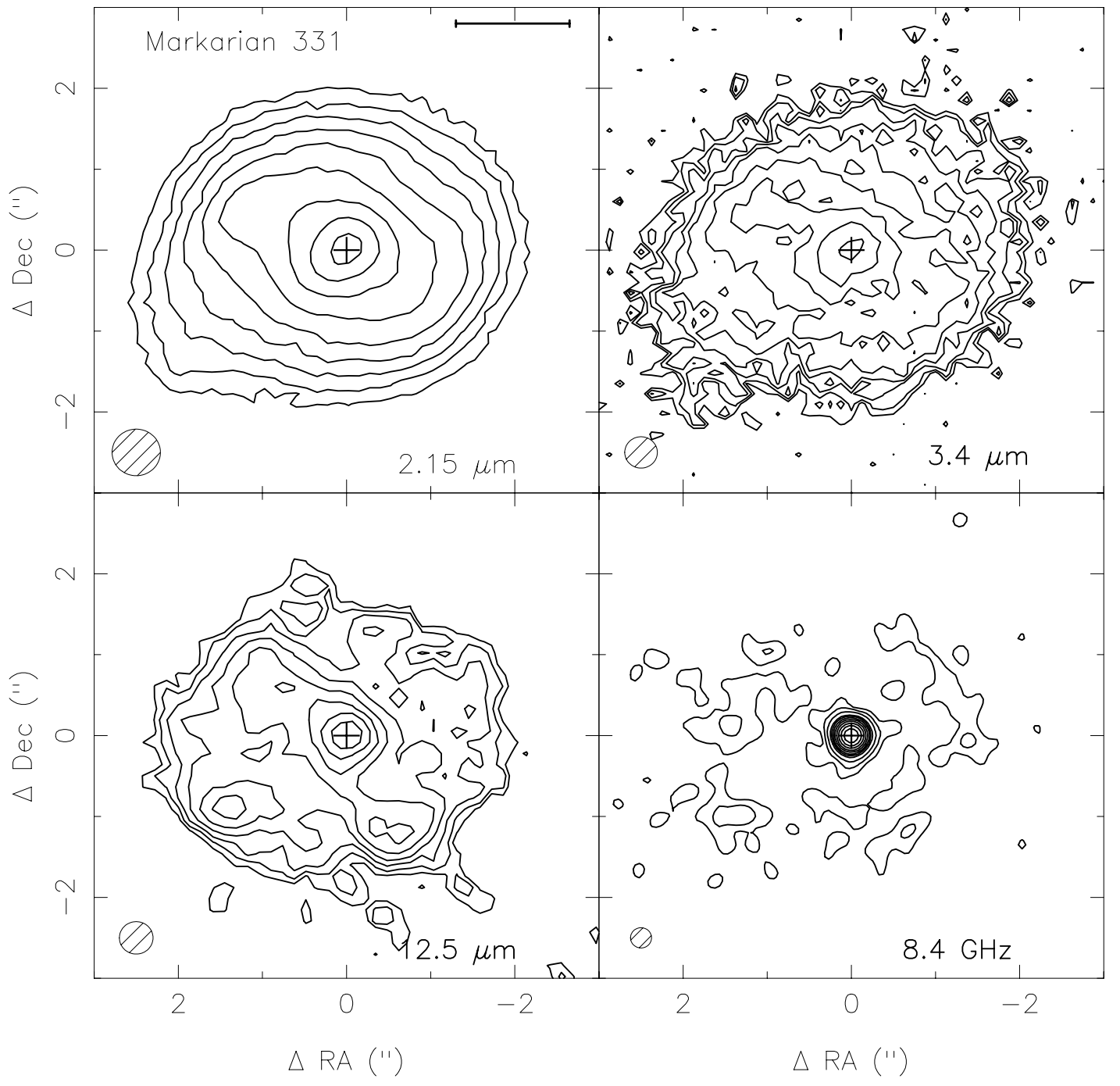

FIG. $7 a$

FIG. 7.-(a) Montage of contour plots of Mrk 331 at $2.15 \mu \mathrm{m}, 3.4 \mu \mathrm{m}, 12.5 \mu \mathrm{m}$, and $8.4 \mathrm{GHz}$. The origin is chosen to be at the bright nucleus in each panel and is indicated by a plus sign. The $2.15 \mu \mathrm{m}$ and $3.4 \mu \mathrm{m}$ images are from the 200 inch Hale telescope. (b) Contour map of Mrk 331 at $12.5 \mu \mathrm{m}$ overlaid on the corresponding gray-scale image of $8.4 \mathrm{GHz}$ emission. (c) Spectral energy distribution of sources in Mrk 331 are shown. The filled circles represent the integrated fluxes taken from IRAS and 2MASS data. The aperture photometry in a $4^{\prime \prime}$ diameter beam is represented by filled squares, the photometry in a $1 . .5$ diameter beam centered on the nucleus is represented by open circles. The photometry in the annulus between the $1 . .5$ diameter and $4^{\prime \prime}$ diameter is shown as open squares. 


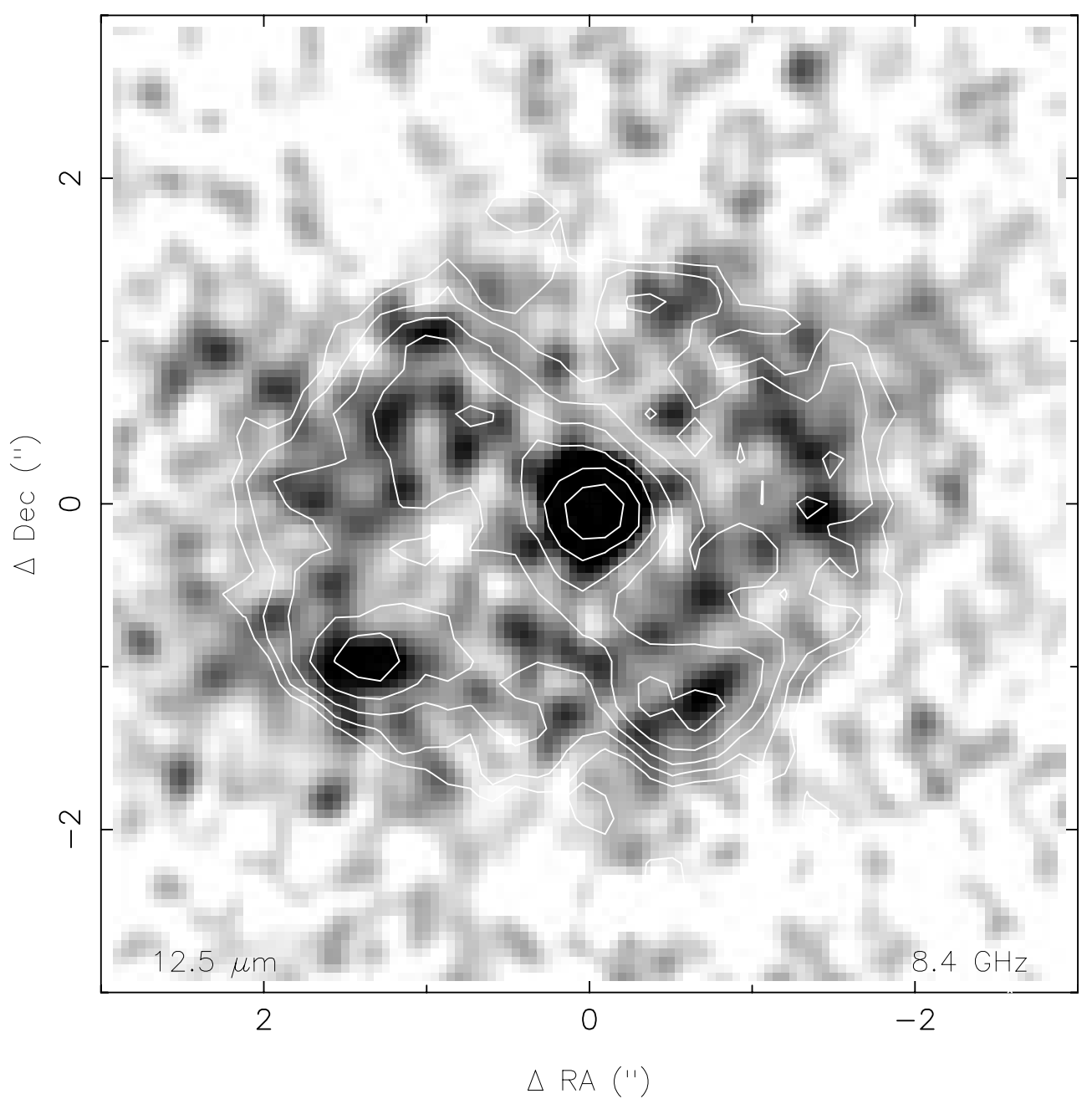

FIG. $7 b$

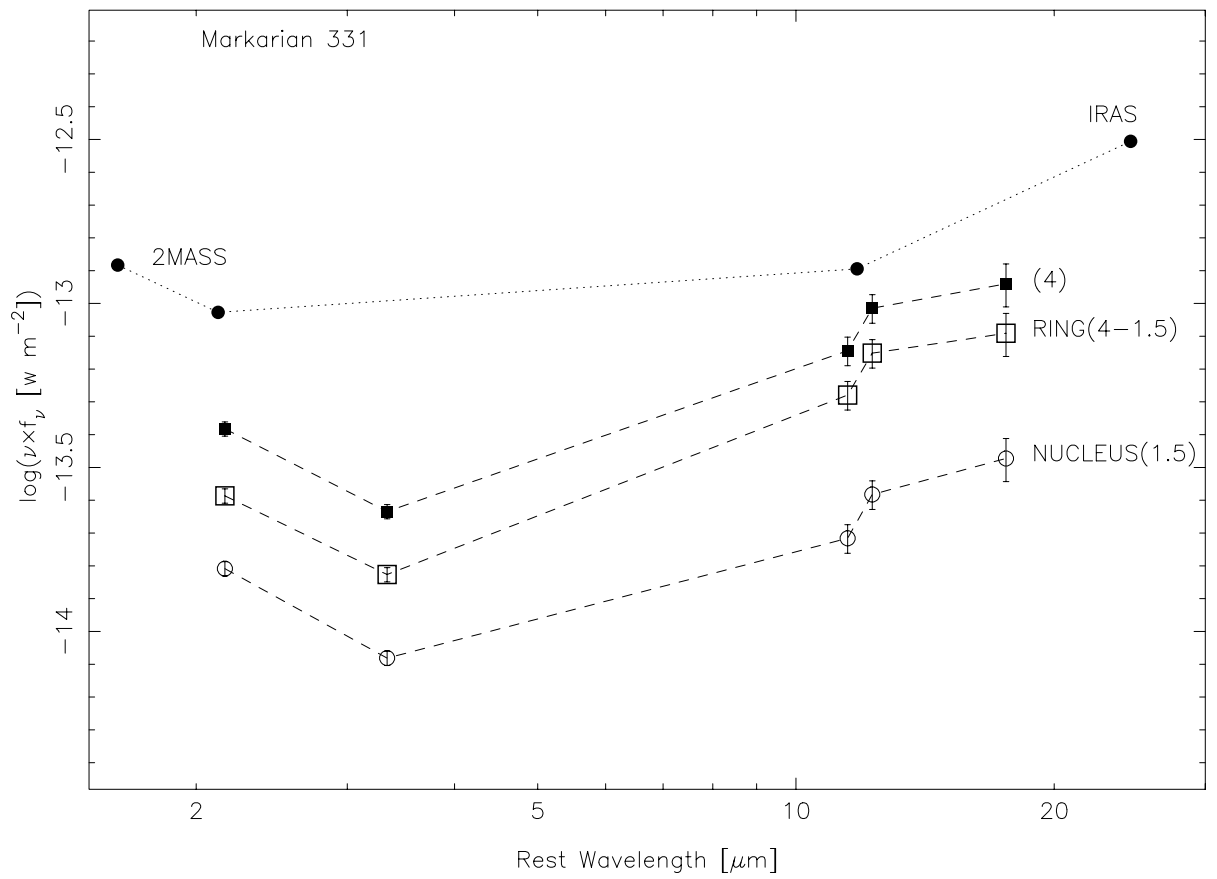

FIG. $7 c$ 
the emission is apparently confined to the nucleus and the ring. The latter effect may be a result of the very high resolution of the VLA image. The structure at $3.4 \mu \mathrm{m}$ appears quite similar to that at $12.5 \mu \mathrm{m}$, showing a compact nucleus, a bar to the northeast, and a disk. Figure $7 b$ compares the images in the mid-infrared and radio directly, with an overlay of the $12.5 \mu \mathrm{m}$ contours on a gray-scale $8.4 \mathrm{GHz}$ image. The structures are quite similar, with the peaks in the radio image corresponding very closely to the features in the mid-infrared image.

Figure $7 c$ plots the fluxes in this system, comparing the mid-infrared flux densities with the IRAS and 2MASS measurements. The ground-based data account for greater than $80 \%$ of the $12 \mu \mathrm{m}$ flux density measured by $I R A S$, showing that roughly the central kiloparsec accounts for nearly all the infrared luminosity in this system. The similarity of the images at 11.7 and $12.5 \mu \mathrm{m}$ shows that there is little evidence for variation in the spectral distribution of the infrared emission over the nuclear region. Roche et al. (1991) show that there is a strong PAH feature in the $10 \mu \mathrm{m} \mathrm{spec}-$ trum of this galaxy, as well as strong silicate absorption.

\section{DISCUSSION}

The galaxies observed in this study were selected to be highly luminous, active star-forming galaxies that are bright in the $12 \mu \mathrm{m} I R A S$ band. The mid-infrared observations reported here generally account for well over half of the total $12 \mu \mathrm{m}$ flux density as measured by IRAS. This is seen in the figures of SEDs of the observed galaxies, where the observed mid-infrared fluxes are plotted with the IRAS fluxes. Only in NGC 6090 and VV 114 is the $12.5 \mu \mathrm{m}$ flux observed here less than half the IRAS $12 \mu \mathrm{m}$ flux. In the remaining five systems, the compact structures observed in the Keck images account for $80 \%-100 \%$ of the total $12 \mu \mathrm{m}$ flux density from the system. Thus our first conclusion is that star formation in these systems is not occurring over the entire disk of the galaxy but is confined to relatively compact regions having sizes of $\sim 100 \mathrm{pc}$ up to $\sim 1 \mathrm{kpc}$.

The mid-infrared emission in these systems, as characterized by the $12.5 \mu \mathrm{m}$ images presented in Figures $1-7$, is far more compact than the overall distribution of near-infrared light in these systems. This can be seen by comparing the $12.5 \mu \mathrm{m}$ images of Figures $1 a-7 a$ with the corresponding large-field $2.2 \mu \mathrm{m}$ NICMOS images from S00. The sizes of the mid-infrared sources are typically $\sim 00^{\prime \prime} 3-2^{\prime \prime}$, while the galaxy sizes seen in the NICMOS images (S00) are typically $10^{\prime \prime}-30^{\prime \prime}$. The significantly smaller mid-infrared sizes compared with the near-infrared sizes of these sources is shown directly in Figure 8, where we present the curves of growth of the $12.5 \mu \mathrm{m}$ flux as a function of beam radius, compared

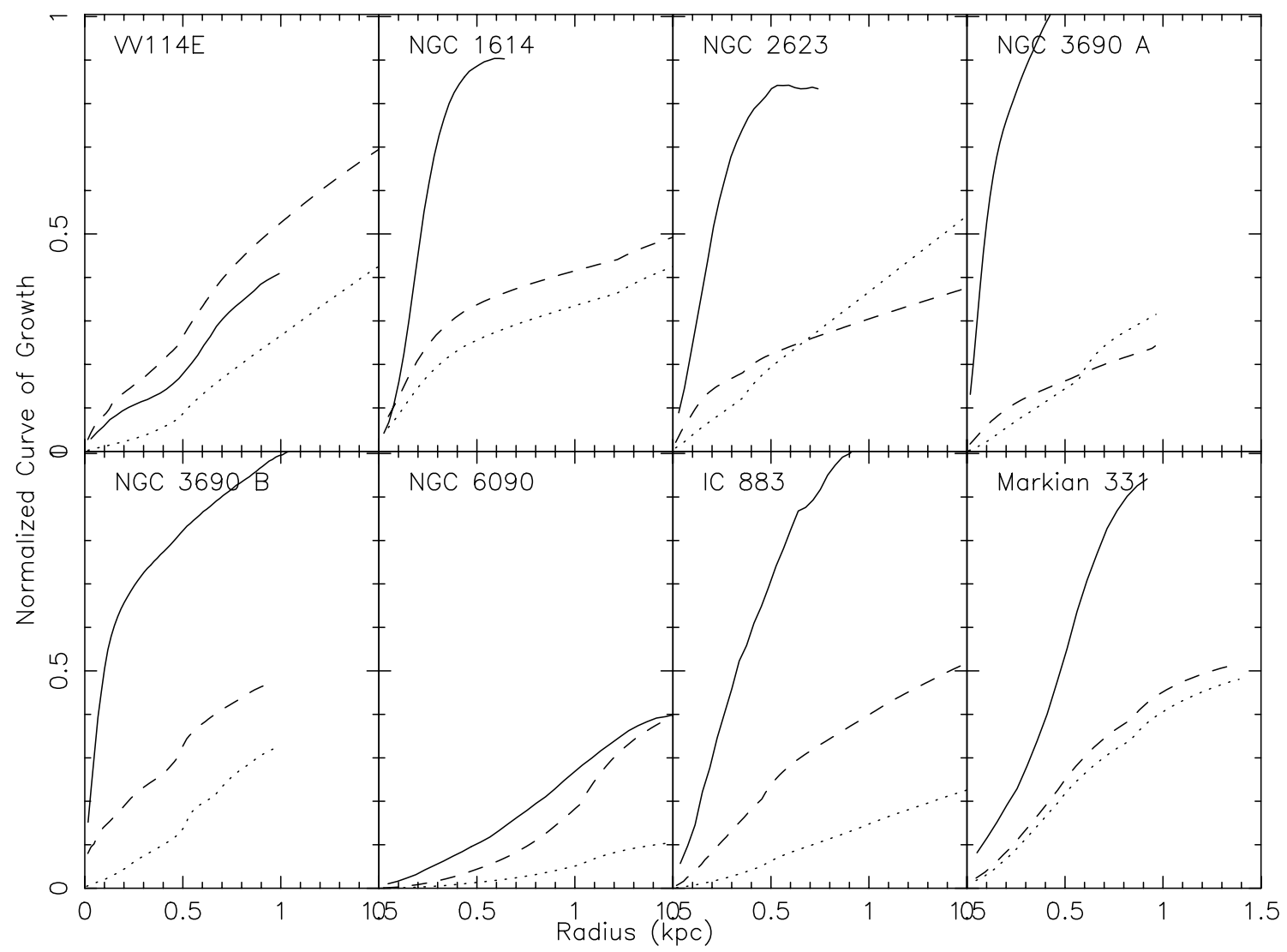

Fig. 8.-Montage of the normalized curves of growth (included flux density vs. beam size) for the galaxies in this sample at $12.5 \mu \mathrm{m}$ (solid line), $2.2 \mu \mathrm{m}$ (dashed line), and $1.1 \mu \mathrm{m}$ (or $1.2 \mu \mathrm{m}$ in the case of Mrk 331, dotted line). The normalization to 1.0 is taken from the IRAS $12 \mu \mathrm{m}$ flux density for the $12.5 \mu \mathrm{m}$ curve and the integrated magnitudes of these galaxies at 2.2 and $1.2 \mu \mathrm{m}$ from the 2MASS all-sky survey (T. Jarrett 2000, private communication). The physical scale for each galaxy was taken from the data in Table 1 . No correction has been applied for the PSF size, so that in some cases, e.g., NGC 3690 A and B and NGC 2623, the profile reflects the curve of growth of the PSF. In all cases the centers of the beams for the curves of growth are at the peak surface brightness at $12.5 \mu \mathrm{m}$, as shown in Figs. 1a-7a. The same location is taken for the center of the beams at all wavelengths. In the case of NGC 3690 the center location of NGC $3690 \mathrm{~B}$ is taken as the location of source B1. The effects of the 4 " chopping amplitude are seen in the $12.5 \mu \mathrm{m}$ curves for NGC 1614 , NGC 2623 , and IC 883. 
with those at $2.2 \mu \mathrm{m}$ and 1.1 (or 1.2) $\mu \mathrm{m}$ for the galaxies in the sample. The $12.5 \mu \mathrm{m}$ flux versus beam size was determined from the images in Figures $1 a-7 a$, with the normalization to $100 \%$ provided by the IRAS measured total flux density at $12 \mu \mathrm{m}$. The origin for the measurement beams was the location of peak $12.5 \mu \mathrm{m}$ brightness as identified in the galaxy images of Figures $1 a-7 a$.

The corresponding curves of growth at 1.1 and $2.2 \mu \mathrm{m}$ were determined from NICMOS images for VV 114, NGC 2623, IC 883, NGC 6090 (from S00), NGC 1614 (from Alonso-Herrero et al. 2001), and NGC 3690/IC 694 (from Alonso-Herrero et al. 2000) and from Palomar $J$ and $K_{s}$ imaging data for Mrk 331. The large beam normalizations for these data were based on the total $J$ and $K$ magnitudes of these galaxies as measured in the 2MASS database (T. Jarrett 2000, private communication). For the 1.1 and 2.2 $\mu \mathrm{m}$ curves of growth, the origin for the measurement beams was the same as for the $12.5 \mu \mathrm{m}$ beams, as shown in Figures $1 a-7 a$.

Figure 8 shows that for the galaxies where the groundbased $12.5 \mu \mathrm{m}$ images account for the bulk of the total 12 $\mu \mathrm{m}$ flux of the galaxy, the radii of the regions producing $50 \%$ of the flux at $12.5 \mu \mathrm{m}$ are less than $100 \mathrm{pc}$ to $\sim 300 \mathrm{pc}$, while the equivalent radii at 1.2 and $2.2 \mu \mathrm{m}$ are 1 to greater than $2 \mathrm{kpc}$. Noticeably different from this general characteristic are VV 114 and NGC 6090, where a substantial fraction $(\sim 60 \%)$ of the $12 \mu \mathrm{m}$ light is not detected in the ground-based observation and is presumably extended, low surface brightness emission distributed over the galaxies. For both VV 114E and NGC 6090 the curves of growth at 2.2 and $12.5 \mu \mathrm{m}$ are quite similar.

The $2.2 \mu \mathrm{m}$ light distribution is, in principle, relatively insensitive to dust extinction, although this is not the case for very dusty environments in the galaxies. To the extent that older stellar populations dominate the near-infrared light in these galaxies, the $2.2 \mu \mathrm{m}$ light traces the mass distribution of that population.

The $12 \mu \mathrm{m}$ emission traces the current sites of luminous star formation. If the star-forming regions within these galaxies are local examples of distant, dusty star-forming galaxies, the global SEDs of the distant star-forming galaxies would be more like those of the starburst regions within these galaxies, rather than like the SEDs of the whole galaxies, which have major contributions in the near-infrared from stars not associated with the current starburst. The difference between the global SEDs and the SEDs of the star-forming regions is illustrated quantitatively in the figures by the comparison of the integrated SEDs with the small aperture SEDs of the individual $12.5 \mu \mathrm{m}$ sources in the galaxies. In all the galaxies, the SED of the individual star-forming regions shows a significant increase in ratio of 12.5 to $2.2 \mu \mathrm{m}$ flux in the small beams, compared with the integrated light of the galaxies. Typically, this ratio is larger by a factor of 2 in the star-forming region as compared with the integrated light for the galaxy, but it ranges to nearly an order of magnitude for the reddest source $\left(\mathbf{C}^{\prime}\right)$ in NGC 3690. For distant star-forming galaxies, such as those seen in SCUBA submillimeter surveys (e.g., Eales et al. 2000), SIRTF observations will be required to obtain the equivalent observations to those presented here.

Presumably, the $12.5 \mu \mathrm{m}$ emission is tracing the farinfrared emission associated with the same star formation complexes. If the fraction of the total infrared luminosity emitted by the individual regions in these galaxies is similar to the fraction of the $12.5 \mu \mathrm{m}$ flux density compared with the total $12 \mu \mathrm{m}$ flux density measured by $I R A S$, these regions produce from $\sim 10^{10} L_{\odot}$ to $\sim 4 \times 10^{11} L_{\odot}$ within a few hundred parsecs. While not as luminous as the ULIRGs, the star formation rates are still prodigious by comparison with normal galaxies such as the Milky Way. The star formation rates are from 0.7 to $30 M_{\odot} \mathrm{yr}^{-1}$, based on the conservative luminosity-star formation rate conversion of Scoville \& Young (1983) or Scoville \& Soifer (1990). If the conversion from luminosity to star formation rate is a factor of several greater than this, as is the case for models that account for the formation of stars with mass less than $1 M_{\odot}$ (see Kennicutt 1998), the total star formation rates go up accordingly.

Since the mid-infrared sources trace the high-luminosity regions in these galaxies, these data provide direct evidence of the physical characteristics of the regions in which the starburst luminosity is being generated. Table 3 presents a summary of the sizes, brightness temperatures, and color temperatures of the mid-infrared sources. The inferred brightness temperature at $60 \mu \mathrm{m}$ is calculated assuming the same angular size as measured at $12.5 \mu \mathrm{m}$ and assuming the same fraction of the total $60 \mu \mathrm{m}$ flux density (as determined from the IRAS data) emerges from the region as is observed for the source at $12 \mu \mathrm{m}$. In physical sizes, the detected sources range from less than $110 \mathrm{pc}$ to almost $1 \mathrm{kpc}$. In a few cases, it is not physically possible for the bulk of the luminosity to emerge from sources of the sizes inferred from the $12.5 \mu \mathrm{m}$ images, since the brightness temperature at 60 $\mu \mathrm{m}$ is larger than the color temperature of the source (here we assume that the color temperature is the global color temperature, determined from the IRAS 60 and $100 \mu \mathrm{m}$ flux densities). In these cases, the far-infrared source size must be larger than the observed $12.5 \mu \mathrm{m}$ source size. The minimum source sizes necessary for equal color and brightness temperatures at $60 \mu \mathrm{m}$ are also given in Table 3, and they generally are close to, or slightly larger than, the upper limits on sources sizes determined from the $12.5 \mu \mathrm{m}$ Keck observations.

With these estimates of the physical sizes of the emitting regions determined, we can compare the derived surface brightnesses with those of both lower and higher luminosity sources. Table 3 includes the surface brightnesses in $L_{\odot}$ $\mathrm{kpc}^{-2}$ derived from the FWHM estimated source sizes (corrected for the PSF size) and the fraction of the total luminosity of the source inferred from the $12.5 \mu \mathrm{m}$ measured flux density for that source. As can be seen from Table 3 , these values range from $2 \times 10^{11}$ to $\sim 2 \times 10^{13} L_{\odot}$ $\mathrm{kpc}^{-2}$. By comparison, the infrared surface brightnesses in the ULIRGs observed by Soifer et al. (2000) range from $1 \times 10^{12}$ to $\sim 6 \times 10^{13} L_{\odot} \mathrm{kpc}^{-2}$. In physical size, the starburst regions range from $\sim 120 \mathrm{pc}$ (in VV $114 \mathrm{E}_{\mathrm{NE}}$ ) to $\sim 1.2$ $\mathrm{kpc}$ (in Mrk 331), while the corresponding sizes of starburst regions in ULIRGs are quite similar, ranging from $140 \mathrm{pc}$ in Arp 220 to $1.6 \mathrm{kpc}$ in IRAS $17208-0014$. Thus, while the apparent physical sizes are similar to those found in ULIRGs, the apparent surface brightnesses of star formation are lower by factors of 3-10 or more. The difference in luminosities between the luminous starburst galaxies and the LUGS appears to be in the luminosity generated in a given volume rather than in the volume in which the luminosity is generated.

The core of Orion, with a luminosity of $2 \times 10^{5} L_{\odot}$ over $0.3 \mathrm{pc}$ (Werner et al. 1976), has an apparent surface bright- 
TABLE 3

Sizes and Surface Brightnesses in Starburst GalaXIES

\begin{tabular}{|c|c|c|c|c|c|c|c|c|}
\hline \multirow[b]{2}{*}{ ОвЈест } & \multicolumn{2}{|c|}{ SIZE OF MID-IR SOURCE } & \multirow{2}{*}{$\frac{f_{12.5 \mu \mathrm{m}}(\mathrm{Keck})}{f_{12 \mu \mathrm{m}}(I R A S)}$} & \multirow{2}{*}{$\begin{array}{c}T_{b}(12 \mu \mathrm{m}) \\
(\mathrm{K})\end{array}$} & \multirow{2}{*}{$\begin{array}{c}T_{b}(60 \mu \mathrm{m}) \\
(\mathrm{K})\end{array}$} & \multirow{2}{*}{$\begin{array}{l}T_{c}(60 \mu \mathrm{m}) \\
\quad(\mathrm{K})\end{array}$} & \multirow[b]{2}{*}{$L_{\mathrm{IR}}\left[L_{\odot}\right]$} & \multirow[b]{2}{*}{$L / A\left[L_{\odot} \mathrm{kpc}^{-2}\right]$} \\
\hline & $(\operatorname{arcsec})$ & (pc) & & & & & & \\
\hline \multirow[t]{2}{*}{ VV 114E sw ......... } & 0.37 & 150 & 0.30 & 94 & 57 & 54 & $1.2 \times 10^{11}$ & \\
\hline & $>0.41$ & $>165$ & 0.30 & 92 & 54 & 54 & $1.2 \times 10^{11}$ & $<5.6 \times 10^{12}$ \\
\hline VV 114E ne ......... & 0.29 & 120 & 0.12 & 91 & 51 & 54 & $5.0 \times 10^{10}$ & $4 \times 10^{12}$ \\
\hline NGC $1614 \ldots \ldots \ldots$. & 1.70 & 550 & 0.87 & 84 & 41 & 62 & $3.6 \times 10^{11}$ & $1.5 \times 10^{12}$ \\
\hline \multirow[t]{2}{*}{ NGC $2623 \ldots \ldots \ldots$} & $0.7 \times<0.3$ & $260 \times<110$ & 0.85 & $>89$ & $>65$ & 64 & $3.0 \times 10^{11}$ & \\
\hline & $>0.52$ & $>192$ & 0.85 & 88 & 64 & 64 & $3.0 \times 10^{11}$ & $<1.0 \times 10^{13}$ \\
\hline \multirow[t]{2}{*}{ NGC $3690 \mathrm{~A} \ldots . . . .}$. & $<0.60$ & $<125$ & 0.32 & $>100$ & 69 & 66 & $2.6 \times 10^{11}$ & \\
\hline & $>0.65$ & $>137$ & 0.32 & 98 & 66 & 66 & $2.6 \times 10^{11}$ & $<1.8 \times 10^{13}$ \\
\hline NGC $3690 \mathrm{C}^{\prime} \ldots . . .$. & $<0.60$ & $<125$ & 0.04 & $>85$ & $>44$ & 66 & $3.2 \times 10^{10}$ & $>2.6 \times 10^{12}$ \\
\hline NGC $3690 \mathrm{C} \ldots . . . .$. & 1.1 & 230 & 0.12 & 79 & 42 & 66 & $1.0 \times 10^{11}$ & $2.4 \times 10^{12}$ \\
\hline \multirow[t]{2}{*}{ NGC 3690 B1 ...... } & $<0.60$ & $<125$ & 0.51 & $>104$ & $>80$ & 66 & $4.0 \times 10^{11}$ & \\
\hline & $>0.84$ & $>176$ & 0.51 & 98 & 66 & 66 & $4.0 \times 10^{11}$ & $<1.6 \times 10^{13}$ \\
\hline NGC 3690 B2 ..... & $<0.6$ & $<125$ & 0.015 & $>78$ & $>37$ & 66 & $1.2 \times 10^{10}$ & $>1.0 \times 10^{12}$ \\
\hline IC $883 \ldots \ldots \ldots \ldots$ & $1.5 \times 0.4$ & $700 \times 190$ & 1.00 & 84 & 20 & 46 & $3.2 \times 10^{11}$ & $2.0 \times 10^{12}$ \\
\hline NGC 6090E S ...... & $1.5 \times 1.0$ & $890 \times 590$ & 0.30 & 73 & 30 & 51 & $9.7 \times 10^{10}$ & $2.4 \times 10^{11}$ \\
\hline NGC 6090E N...... & 1.0 & 590 & 0.12 & 71 & 28 & 51 & $3.9 \times 10^{10}$ & $1.4 \times 10^{11}$ \\
\hline NGC 6090W ........ & $<0.6$ & $<350$ & 0.04 & 71 & $>29$ & 51 & $1.3 \times 10^{10}$ & $>1.3 \times 10^{11}$ \\
\hline Mrk $331 \mathrm{n} \ldots \ldots \ldots \ldots$ & $(<0.6)$ & $<225$ & 0.2 & $>81$ & $>41$ & 58 & $5 \times 10^{10}$ & $>1.3 \times 10^{12}$ \\
\hline Mrk 331 r ........... & $4 \times 2.7$ & $1500 \times 1000$ & 0.63 & 71 & 30 & 58 & $1.6 \times 10^{11}$ & $1.4 \times 10^{11}$ \\
\hline
\end{tabular}

ness of $3 \times 10^{12} L_{\odot} \mathrm{kpc}^{-2}$, while the apparent face-on surface brightness of the starburst region in M82 is $\sim 2 \times 10^{11} L_{\odot} \mathrm{kpc}^{-2}$ (Soifer et al. 2000). Previous work (Meter et al. 1997) has shown that in normal galaxies, the surface brightness of star formation reaches an upper limit of $\sim 2 \times 10^{11} L_{\odot} \mathrm{kpc}^{-2}$ globally averaged over entire galaxies, with peak surface brightnesses of clusters of size $\sim 10$ pc of $\sim 5 \times 10^{13} L_{\odot} \mathrm{kpc}^{-2}$. Thus the starburst regions in these systems appear extraordinary in comparison with normal galaxies, but not with respect to the highest density star-forming environments within galaxies.

Table 4 summarizes the surface brightnesses of nearby starburst systems, the starburst regions in the galaxies in this sample, and the starburst regions in ULIRGs from Soifer et al. (2000). We can see clearly here that, while there is significant scatter, there appears to be a natural progression in surface brightness in the galaxies, with increasing surface brightness accompanying increasing luminosity. Table 4 shows that in these luminous starburst galaxies

TABLE 4

Surface Brightnesses of Infrared Starburst GalaXies

\begin{tabular}{clcr}
\hline \hline Object & \multicolumn{1}{c}{ Type } & $\begin{array}{c}\text { Infrared } \\
\text { Luminosity } \\
\left(L_{\text {bol }}\left(L_{\odot}\right)\right.\end{array}$ & $\begin{array}{r}\text { Surface } \\
\text { Brightness } \\
\left(L_{\odot} \mathrm{kpc}^{-2}\right)\end{array}$ \\
\hline Orion $\ldots \ldots \ldots$. & H II region & $1 \times 10^{6}$ & $2 \times 10^{12}$ \\
M $82 \ldots \ldots \ldots$ & Local starburst & $3 \times 10^{10}$ & $2 \times 10^{11}$ \\
NGC $6090 \ldots$. & Starburst & $3 \times 10^{11}$ & $2 \times 10^{11}$ \\
NGC $1614 \ldots$. & Starburst & $4 \times 10^{11}$ & $1.5 \times 10^{12}$ \\
Mrk $331 \ldots \ldots$ & Starburst & $2.5 \times 10^{11}$ & $\sim 2 \times 10^{12}$ \\
IC $883 \ldots \ldots \ldots$ & Starburst & $3 \times 10^{11}$ & $2 \times 10^{12}$ \\
VV $114 \ldots \ldots$. & Starburst & $4 \times 10^{11}$ & $\sim 5 \times 10^{12}$ \\
NGC $2623 \ldots$. & Starburst & $3 \times 10^{11}$ & $\sim 10^{13}$ \\
NGC $3690 \ldots$. & Starburst & $8 \times 10^{11}$ & $\sim 10^{13}$ \\
IRAS $17208 \ldots$ & ULIRG & $3 \times 10^{12}$ & $1.2 \times 10^{12}$ \\
Mrk 273 $\ldots \ldots$. & ULIRG & $1.3 \times 10^{12}$ & $>2.2 \times 10^{13}$ \\
IRAS $08572 \ldots$ & ULIRG & $1.3 \times 10^{12}$ & $>2.8 \times 10^{13}$ \\
Arp $220 \ldots \ldots$. & ULIRG & $1.5 \times 10^{12}$ & $6.0 \times 10^{13}$ \\
\hline
\end{tabular}

environments equivalent to the core of Orion extend over hundreds of parsecs.

Turner, Beck, \& Ho (2000) and Gorjian, Turner, \& Beck (2001) have recently shown that in the nearby dwarf galaxy NGC 5253 star formation that produces $\sim 2 \times 10^{9} L_{\odot}$ is occurring in a region $\sim 2 \mathrm{pc}$ in size, i.e., in a "super-star cluster." The observations described here cannot rule out the possibility that much of the luminosity in the starburst galaxies studied here emerges from regions of similar size, although the most luminous systems in this sample would require clusters of $\sim 100$ such super-star clusters in regions of order $\sim 200 \mathrm{pc}$ in diameter.

In about half of the galaxies in this sample, the luminosity appears to be generated in nuclear starbursts. In NGC 1614 and Mrk 331, a substantial fraction of the infrared luminosity is generated in a ring surrounding the nucleus. NGC 6090 presents the greatest exception to the nuclear starburst picture for high-luminosity systems. Here the luminosity appears to be generated where the molecular gas remains from the recent interaction between the two galaxies. This might be the best example for a starburst triggered by cloud-cloud collisions in the galaxies disks.

The mid-infrared and radio emission generally trace each other quite well. Differences at the $100 \mathrm{pc}$ scale are seen in VV $114 \mathrm{E}_{\mathrm{Sw}}$ and in the more extended emission in NGC 6090 and IC 883. It is most likely that the differences in the diffuse emission is a lack of sensitivity to low surface brightness emission in the $8.4 \mathrm{GHz}$ data.

\section{SUMMARY AND CONCLUSIONS}

We have obtained mid-infrared diffraction limited imaging of seven starburst galaxies with the Keck telescopes. These observations have shown the following:

1. A substantial fraction, usually more than $50 \%$, of the mid-infrared luminosity in these systems is generated in regions ranging in size from $\sim 100 \mathrm{pc}$ to $1 \mathrm{kpc}$.

2. Nuclear starbursts generally dominate the starburst activity in these galaxies. 
3. In some cases, most notably NGC 6090 , substantial infrared luminosity is generated in regions away from the galaxy nuclei and is more likely associated with the region of physical interaction between two galaxies.

4. The radio emission in starburst galaxies is a good tracer of the current location of compact star-forming regions.

5. Mid-infrared emission is much more compact than is near-infrared emission in these galaxies. If the starburst regions in these galaxies are prototypes for star-forming galaxies at high redshift, the energy output of such galaxies should be dominated by the mid- and far-infrared energy output more than is the case in the integrated light emerging from nearby starburst galaxies.

We thank J. Aycock, T. Stickel, G. Wirth, R. Campbell, and Lee Armus for assistance with the observations. Barbara Jones, Rick Puetter, and the Keck team brought the long wavelength spectrograph (LWS) into service, enabling many of these observations. We thank Marcia Rieke and Susan Neff for providing data for NGC 3690,
6090, and 1614. Vassilis Charmandaris provided advice about ISO observations of VV 114. Joe Mazzarella provided us with data from NED, Tom Jarrett provided data from 2MASS, and Aaron Evans provided images from NICMOS. We also thank an anonymous referee for critical comments on this paper. This research has made use of the NASA/IPAC Extragalactic Database (NED) which is operated by the Jet Propulsion Laboratory, California Institute of Technology, under contract with the National Aeronautics and Space Administration. B. T. S., G. N., K. M., and E. E. are supported by grants from the NSF and NASA. B. T. S. and S. S. are supported by the SIRTF Science Center at Caltech. SIRTF is carried out at the Jet Propulsion Laboratory. N. Z. S. was supported by NASA grant NAG 5-3042. This work was carried out in part (M. R.) at the Jet Propulsion Laboratory, which is operated by the California Institute of Technology under agreement with NASA. The development of MIRLIN was supported by NASA's Office of Space Science. The National Radio Astronomy Observatory (J. J. C.) is a facility of the National Science Foundation operated under cooperative agreement by the Associated Universities for Research in Astronomy, Inc.

\section{REFERENCES}

Alonso-Herrero, A., Engelbracht, C. W., Rieke, M. J., Rieke, G. H., \& Quillen, A. C. 2001, ApJ, 546, 952

Alonso-Herrero, A., Rieke, G. H., Rieke, M. J., \& Scoville, N. Z. 2000, ApJ, 532,845

Arp, H. 1966, ApJS, 14, 1

Beichman, C. A., Neugebauer, G., Habing, H. J., Clegg, P. E., \& Chester, T. J., ed. 1989, IRAS Catalog and Atlases, Explanatory Supplement (2d ed.; Washington: GPO)

Bryant, P. M., \& Scoville, N. Z. 1999, 117, 2632

Carico, D. P., Sanders, D. B., Soifer, B. T., Elias, J. H., Matthews, K., \& Neugebauer, G. 1988, AJ, 95, 356

Condon, J. J., Helou, G., Sanders, D. B., \& Soifer, B. T. 1990, ApJS, 73, 359

Condon, J. J., Huang, Z. P., Yin, Q. F., \& Thuan, T. X. 1991, ApJ, 378, 65 (C91)

Dale, D. A., Helou, G., Contursi, A., Silbermann, N. A., \& Sonali, K. 2001, ApJ, 549, 215

Dinshaw, N. Evans, A. S., Epps, H., Scoville, N. Z., \& Rieke, M. J. 1999, ApJ, 525, 702

Doyon, R., Nadeau, D, Joseph, R. D., Goldader, J. D., Sanders, D. B., \& Rowlands, N. 1995, ApJ, 450, 111

Dudley, C. C. 1999, MNRAS, 307, 553

Eales, S., Simon, L., Webb, T., Dunne, L., Gear, W., Clements, D., \& Yun, M. 2000, AJ, 120, 2244

Frayer, D. T., Ivison, R. J., Smail, I., Yun, M. S., \& Armus, L. 1999, AJ, 118, 139

Gehrz, R. D., Sramek, R. A., \& Weedman, D. W. 1983, ApJ, 267, 551

Gorjian, V., Turner, J. L., \& Beck, S. C. 2001, ApJ, 554, L29

Harper, D. A., \& Low, F. J. 1973, ApJ, 182, L89

Hauser, M. G., et al. 1998, ApJ, 508, 25

IRAS Point Source Catalog, Version 2. 1989, Joint IRAS Science Working Group (Washington: GPO)

Jones, B., \& Puetter, R. C. 1993, Proc. SPIE, 1946, 610

Kennicutt, R. C. 1998, ApJ, 498, 541

Keto, E., et al. 1997, ApJ, 485, 598

Kim, D.-C., Sanders, D. B., Veilleux, S., Mazzarella, J. M., \& Soifer, B. T. 1995, ApJS, 98, 129

Kleinmann, D. E., \& Low, F. J. 1970, ApJ, 161, L203

Knop, R. A., Soifer, B. T., Graham, J. R., Matthews, K., Sanders, D. B., \& Scoville, N. Z. 1994, AJ, 107, 920

Laurent, O., Mirabel, I. F., Charmandaris, V., Gallais, P., Madden, S. C., Sauvage, M., Vigroux, L., \& Cesarsky, C. 2000, A\&A, 359, 887

Li, A., \& Draine, B. T. 2001, ApJ, 554, 778

Matthews, K., \& Soifer, B. T. 1994, Infrared Astronomy with Arrays: The Next Generation, ed. I. McLean (Dordrecht: Kluwer), 239
Meurer, G. R., Heckman, T. M., Lehnert, M. D., Leitherer, C., \& Lowenthal, J. 1997, AJ, 114, 54

Miles, J. W., Houck, J. R., Hayward, T. L., \& Ashby, M. L. N. 1996, ApJ, 465, 191

Murphy, T. W., Soifer, B. T., \& Matthews, K. 1999, PASP, 111, 1176

Neff, S. G., Hutchings, J. B., Stanford, S. A., \& Unger, S. W. 1990, AJ, 99, 1088

Puget, J. L., Abergel, A., Bernard, J. P., Boulanger, F., Desert, F. X., \& Hartmann, D. 1996, A\&A, 308, L5

Ressler, M. E., Werner, M. W., Van Cleve, J., \& Choa, H. 1994, Exp. Astron., 3, 277

Rieke, G. H., \& Low, F. J. 1972, ApJ, 176, L95

Roche, P. F., Aitken, D. K., Smith, C. H., \& Ward, M. J. 1991, MNRAS, 248,606

Sanders, D. B., \& Mirabel, I. F. 1996, ARA\&A, 34, 749

Sanders, D. B., Soifer, B. T., Elias, J. H., Madore, B. F., Matthews, K., Neugebauer, G., \& Scoville, N. Z. 1988, ApJ, 325, 74

Sargent, A. I., \& Scoville, N. Z. 1991, ApJ, 366, L1

Satyapal, S., Watson, D. M., Forrest, W. J., Pipher, J. L., Fischer, J., Greenhouse, M. A., Smith, H. A., \& Woodward, C. E. 1999, ApJ, 516, 704

Scoville, N. Z., et al. 2000, AJ, 119, 991 (S00)

Scoville, N. Z., \& Young, J. S. 1983, ApJ, 265, 148

. 1990, Massive Stars in Starbursts, ed. C. Leitherer, N. Walborn, T. Heckman, \& C. Norman (Cambridge: Cambridge Univ. Press), 233

Soifer, B. T., Boehmer, L., Neugebauer, G., \& Sanders, D. B. 1989, AJ, 98, 766

Soifer, B. T., \& Neugebauer, G. 1991, AJ, 101, 354

Soifer, B. T., et al. 2000, AJ, 119, 509

.2001, in preparation

Soifer, B. T., Sanders, D. B., Madore, B. F., Neugebauer, G., Danielson, G. E., Elias, J. H., Lonsdale, C. J., \& Rice, W. L. 1987, ApJ, 320, 238

Sugai, H., Davies, R. I., Malkan, M. A., McLean, I. S., Usuda, T., \& Ward, M. J. 1999, ApJ, 527, 778

Turner, J. L., Beck, S. C., \& Ho, P. T. P. 2000, ApJ, 532, L109

Veilleux, S., Kim, D.-C., Sanders, D. B., Mazzarella, J. M., \& Soifer, B. T. 1995, ApJS, 98, 171

Werner, M. W., Gatley, I., Becklin, E. E., Harper, D. A., Loewenstein, R. F., Telesco, C. M., \& Thronson, H. A. 1976, ApJ, 204, 420

Wynn-Williams, C. G., Eales, S. A., Becklin, E. E., Hodapp, K.-W., Joseph, R. D., McLean, I. S., Simons, D. A., \& Wright, G. S. 1991, ApJ, 377, 426

Yun, M. S., Scoville, N. Z., \& Knop, R. A. 1994, ApJ, 430, L109 